\title{
The double Riemann zeta function
}

\author{
Hirotaka Akatsuka
}

In 1992 Deninger showed a version of explicit formulas for the Riemann zeta function. In this paper, we establish a duplication of Deninger's explicit formula in the sense of the absolute tensor product due to Kurokawa. As an application, we obtain an Euler product expression for the double Riemann zeta function constructed from the absolute tensor product of the Riemann zeta function.

\section{Introduction}

The prime numbers are closely related to the zeros and the pole of the Riemann zeta function $\zeta(s)$. This relation is described by explicit formulas, which are equations between sums over the prime numbers and sums over the zeros and the pole of the Riemann zeta function. At present, various explicit formulas are known. We refer to Weil [20] as a famous explicit formula. In 1992 Deninger [7] showed the following explicit formula:

Deninger's Theorem 1.1 [7, p. 148]. In $\operatorname{Re}(s)>1$ and $\operatorname{Re}(w)>1$, we have

$$
\begin{aligned}
& \sum_{\rho} \frac{1}{(s-\rho)^{w}}-\frac{1}{(s-1)^{w}}+\sum_{n=1}^{\infty} \frac{1}{(s+2 n)^{w}} \\
& \quad=-\frac{1}{\Gamma(w)} \sum_{p} \sum_{m=1}^{\infty} p^{-m s}(m \log p)^{w-1} \log p
\end{aligned}
$$

where $\rho$ runs over the nontrivial zeros of the Riemann zeta function counted with multiplicity, $\alpha^{w}:=\exp (w \log \alpha), \log \alpha:=\log |\alpha|+\mathrm{i} \arg \alpha$ with $\arg \alpha \in$ $(-\pi / 2, \pi / 2)$ and $p$ runs over the prime numbers.

Remark 1.1. Schröter and Soulé $\left[18\right.$, Theorem, (i)] showed that $\sum_{\rho}(s-$ $\rho)^{-w}$ has a meromorphic continuation to $(w, s) \in \mathbb{C} \times \Omega$, where $\Omega:=\mathbb{C} \backslash$ $\{\rho-\lambda: 0<\operatorname{Re}(\rho)<1, \zeta(\rho)=0, \lambda \geq 0\}$ and $|\arg (s-\rho)|<\pi$. 
In 1992 Kurokawa [11] predicted that for fixed $r \in \mathbb{Z}_{\geq 1}$ there would be a relation between the $r$-ple prime numbers $\left(p_{1}, \ldots, p_{r}\right)$ and the sums $\rho_{1}+\cdots+\rho_{r}$ of the zeros or the pole $\rho_{j}$ of the Riemann zeta function. Under this prediction, he defined the absolute tensor product. Roughly speaking, for meromorphic functions $Z_{1}(s), \ldots, Z_{r}(s)$, their absolute tensor product $\left(Z_{1} \otimes \cdots \otimes Z_{r}\right)(s)$ is defined by the zeta-regularized product (explained later) so as to have zeros or poles at $s=\rho_{1}+\cdots+\rho_{r}$ with $Z_{j}\left(\rho_{j}\right)=0$ or $\infty$ $(j=1, \ldots, r)$ and $\operatorname{Im}\left(\rho_{1}\right), \ldots, \operatorname{Im}\left(\rho_{r}\right)$ having the same signature. The above prediction says that an $r$-ple Riemann zeta function $\zeta^{\otimes r}(s)$ might have a generalization of the Euler product running over the $r$-ple prime numbers $\left(p_{1}, \ldots, p_{r}\right)$ and that there might exist explicit formulas for $\zeta^{\otimes r}(s)$. See $[12$, Section 1] for the precise definition of the absolute tensor product and its expectations. We refer to an excellent survey [15] by Manin. See also Schröter [17] for the study of the absolute tensor product in the name of the Kurokawa tensor product. We also remark that Koyama and Kurokawa [13] obtained an Euler product expression and a Weil's-type explicit formula for $\zeta^{\otimes 2}(s)$. Concerning the absolute tensor product for Euler factors of the Riemann zeta function, see [1-4], [11-14].

The purpose of this paper is to establish a duplication of Deninger's explicit formula in the sense of the absolute tensor product as follows:

Theorem 1.1. In $\operatorname{Re}(s)>2$ and $\operatorname{Re}(w)>2$ we have

$$
\begin{aligned}
& -\sum_{\operatorname{Im}\left(\rho_{1}\right)>0} \sum_{\operatorname{Im}\left(\rho_{2}\right)>0} \frac{1}{\left(s-\rho_{1}-\rho_{2}\right)^{w}}+\sum_{\operatorname{Im}\left(\rho_{1}\right)<0} \sum_{\operatorname{Im}\left(\rho_{2}\right)<0} \frac{1}{\left(s-\rho_{1}-\rho_{2}\right)^{w}} \\
& +2 \sum_{\operatorname{Im}(\rho)>0} \frac{1}{(s-1-\rho)^{w}}-2 \sum_{\operatorname{Im}(\rho)>0} \sum_{n=1}^{\infty} \frac{1}{(s-\rho+2 n)^{w}}-\frac{1}{(s-2)^{w}} \\
& -\sum_{n_{1}=1}^{\infty} \sum_{n_{2}=1}^{\infty} \frac{1}{\left(s+2 n_{1}+2 n_{2}\right)^{w}}+2 \sum_{n=1}^{\infty} \frac{1}{(s+2 n-1)^{w}} \\
& =\frac{1}{\Gamma(w)} \sum_{j=1}^{9} E^{(j)}(w, s),
\end{aligned}
$$

where $\rho, \rho_{1}, \rho_{2}$ run over the nontrivial zeros of the Riemann zeta function in the given ranges counted with multiplicity, the argument lies in $(-\pi / 2, \pi / 2)$ 
and

$$
\begin{aligned}
& E^{(1)}(w, s):=-\frac{s-2}{2 \pi \mathrm{i}} \sum_{p} \sum_{m=1}^{\infty} p^{-m s}(m \log p)^{w-1} \log ^{2} p \\
& +\frac{w+1}{2 \pi \mathrm{i}} \sum_{p} \sum_{m=1}^{\infty} p^{-m s}(m \log p)^{w-2} \log ^{2} p, \\
& E^{(2)}(w, s):=\frac{1}{\pi \mathrm{i}} \sum_{p} \sum_{m=1}^{\infty} \sum_{q} \sum_{\substack{n=1 \\
q^{n} \neq p^{m}}}^{\infty} \frac{p^{-m(s-1)} q^{-n}(m \log p)^{w} \log p}{n(m \log p-n \log q)}, \\
& E^{(3)}(w, s):=-\frac{1}{\pi \mathrm{i}} \sum_{p} \sum_{m=1}^{\infty} \sum_{q} \sum_{n=1}^{\infty} \frac{p^{-m s} q^{-n}(m \log p)^{w} \log p}{n(m \log p+n \log q)}, \\
& E^{(4)}(w, s):=\left(\frac{1}{2}+\frac{\gamma+\log (2 \pi)}{\pi \mathrm{i}}\right)\left(\sum_{p} \sum_{m=1}^{\infty} p^{-m s}(m \log p)^{w-1} \log p\right. \\
& \left.+\sum_{p} \sum_{m=1}^{\infty} p^{-m s}(m \log p)^{w-2} \log p\right) \\
& E^{(5)}(w, s):=\frac{1}{\pi \mathrm{i}} \sum_{p} \sum_{m=1}^{\infty} p^{-m s}(m \log p)^{w-1} \log p \times \frac{\Gamma^{\prime}}{\Gamma}\left(\frac{m \log p}{\pi \mathrm{i}}\right), \\
& E^{(6)}(w, s):=-\frac{1}{\pi \mathrm{i}} \int_{0}^{1}\left(\sum_{p} \sum_{m=1}^{\infty} p^{-m(s-u)}(m \log p)^{w} \log p\right) \log |\zeta(u)| d u, \\
& E^{(7)}(w, s):=-\frac{1}{\pi \mathrm{i}} \int_{0}^{\infty} \frac{u}{\mathrm{e}^{u}-1}\left(\sum_{p} \sum_{m=1}^{\infty} \frac{p^{-m s}(m \log p)^{w-2} \log p}{u+m \log p}\right) d u \\
& E^{(8)}(w, s):=-\sum_{p} \sum_{m=1}^{\infty} p^{-m(s-1)}(m \log p)^{w-1} \log p \\
& E^{(9)}(w, s):=2 \sum_{p} \sum_{m=1}^{\infty} \sum_{k=1}^{\infty} p^{-m(s+2 k)}(m \log p)^{w-1} \log p .
\end{aligned}
$$

Here $p$ and $q$ run over the prime numbers and $\gamma$ is the Euler constant.

Concerning the convergence of the sums and the integrals in $E^{(j)}(w, s)$, the following assertions hold:

Theorem 1.2. (1) For any $j \in\{2,6,8\}$ the sums and the integral in $E^{(j)}$ $(w, s)$ converge absolutely and uniformly on any compact subset of $\left\{(w, s) \in \mathbb{C}^{2}: \operatorname{Re}(s)>2\right\}$. 
(2) For any $j \in\{1,3,4,5,7\}$ the sums and the integral in $E^{(j)}(w, s)$ converge absolutely and uniformly on any compact subset of $\left\{(w, s) \in \mathbb{C}^{2}\right.$ : $\operatorname{Re}(s)>1\}$.

(3) The sum in $E^{(9)}(w, s)$ converges absolutely and uniformly on any compact subset in $\left\{(w, s) \in \mathbb{C}^{2}: \operatorname{Re}(s)>-1\right\}$.

Using Deninger's Theorem 1.1, Deninger obtained a zeta-regularized product expression for $\zeta(s)$. To explain this, we recall the zeta-regularized product. For complex sequences $\mathbf{a}:=\left\{a_{n}\right\}_{n=1}^{\infty}$, the zeta-regularized product is defined by

$$
\prod_{n=1}^{\infty}\left(s-a_{n}\right):=\exp \left[-\operatorname{Res}_{w=0} \frac{Z_{\mathbf{a}}(w, s)}{w^{2}}\right]
$$

with $Z_{\mathbf{a}}(w, s):=\sum_{n=1}^{\infty}\left(s-a_{n}\right)^{-w}$, provided that the Dirichlet series in $Z_{\mathbf{a}}(w, s)$ converges absolutely for sufficiently large $\operatorname{Re}(w)$ and $Z_{\mathbf{a}}(w, s)$ has a meromorphic continuation with respect to $w$ to $\operatorname{Re}(w)>-\varepsilon$ for some $\varepsilon>0$. The important properties are that (1.1) has an analytic continuation to all $s \in \mathbb{C}$ and that its zeros are located at $s=a_{n}(n=1,2, \ldots)$ and nowhere else. Concerning the zeta-regularized product, see [9, 10]. From Deninger's Theorem 1.1, Deninger proved

Deninger's Theorem 1.2. In $\operatorname{Re}(s)>1$ we have

$$
\zeta(s)=\frac{\prod_{\rho}(s-\rho) \prod_{n=1}^{\infty}(s+2 n)}{s-1} .
$$

Remark 1.2. Strictly speaking, Deninger treated $\coprod_{\rho}\left(\frac{s-\rho}{2 \pi}\right)$. See $[7,(3.3)$ Theorem].

Remark 1.3. From Remark 1.1, Deninger's Theorem 1.2 is valid for any $s \in \Omega$. See [18, Theorem, (ii)].

As an application of Theorem 1.1, we show an Euler product expression for $\zeta^{\otimes 2}(s)$. By the definition of the absolute tensor product $\zeta^{\otimes 2}(s)$ is given 
as follows:

$$
\zeta^{\otimes 2}(s)=\frac{\prod_{\operatorname{Im}\left(\rho_{j}\right)>0}\left(s-\rho_{1}-\rho_{2}\right)\left(\prod_{\substack{\operatorname{Im}(\rho)>0 \\ n \geq 1}}(s-\rho+2 n)\right)^{2}(s-2) \prod_{n_{j} \geq 1}\left(s+2 n_{1}+2 n_{2}\right)}{\prod_{\operatorname{Im}\left(\rho_{j}\right)<0}\left(s-\rho_{1}-\rho_{2}\right)\left(\prod_{\operatorname{Im}(\rho)>0}(s-1-\rho)\right)^{2}\left(\prod_{n \geq 1}(s+2 n-1)\right)^{2}} .
$$

We remark that $\zeta^{\otimes 2}(s)$ is given by determining locations and orders of zeros and poles, and that existence and a form of an Euler product expression for $\zeta^{\otimes 2}(s)$ are not trivial. From Theorem 1.1 it is given by the following:

Theorem 1.3. In $\operatorname{Re}(s)>2$ we have

$$
\zeta^{\otimes 2}(s)=\exp \left[\sum_{j=1}^{9} E^{(j)}(s)\right],
$$

where $E^{(j)}(s):=E^{(j)}(0, s)$, that is,

$$
\begin{aligned}
& E^{(1)}(s):=-\frac{s-2}{2 \pi \mathrm{i}} \sum_{p} \sum_{m=1}^{\infty} \frac{p^{-m s} \log p}{m}+\frac{1}{2 \pi \mathrm{i}} \sum_{p} \sum_{m=1}^{\infty} \frac{p^{-m s}}{m^{2}}, \\
& E^{(2)}(s):=\frac{1}{\pi \mathrm{i}} \sum_{p} \sum_{m=1}^{\infty} \sum_{q} \sum_{\substack{n=1 \\
q^{n} \neq p^{m}}}^{\infty} \frac{p^{-m(s-1)} q^{-n} \log p}{n(m \log p-n \log q)}, \\
& E^{(3)}(s):=-\frac{1}{\pi \mathrm{i}} \sum_{p} \sum_{m=1}^{\infty} \sum_{q} \sum_{n=1}^{\infty} \frac{p^{-m s} q^{-n} \log p}{n(m \log p+n \log q)}, \\
& E^{(4)}(s):=\left(\frac{1}{2}+\frac{\gamma+\log (2 \pi)}{\pi \mathrm{i}}\right)\left(\sum_{p} \sum_{m=1}^{\infty} \frac{p^{-m s}}{m}+\sum_{p} \sum_{m=1}^{\infty} \frac{p^{-m s}}{m^{2} \log p}\right), \\
& E^{(5)}(s):=\frac{1}{\pi \mathrm{i}} \sum_{p} \sum_{m=1}^{\infty} \frac{p^{-m s}}{m} \times \frac{\Gamma^{\prime}}{\Gamma}\left(\frac{m \log p}{\pi \mathrm{i}}\right), \\
& E^{(6)}(s):=\frac{1}{\pi \mathrm{i}} \int_{0}^{1} \frac{\zeta^{\prime}}{\zeta}(s-u) \log |\zeta(u)| d u \\
& E^{(7)}(s):=-\frac{1}{\pi \mathrm{i}} \int_{0}^{\infty} \frac{u}{\mathrm{e}^{u}-1}\left(\sum_{p} \sum_{m=1}^{\infty} \frac{p^{-m s} \log p}{(m \log p)^{2}(u+m \log p)}\right) d u
\end{aligned}
$$




$$
\begin{aligned}
& E^{(8)}(s):=-\sum_{p} \sum_{m=1}^{\infty} \frac{p^{-m(s-1)}}{m}, \\
& E^{(9)}(s):=2 \sum_{p} \sum_{m=1}^{\infty} \sum_{k=1}^{\infty} \frac{p^{-m(s+2 k)}}{m} .
\end{aligned}
$$

Remark 1.4. Though Koyama-Kurokawa obtained an Euler product expression for $\zeta^{\otimes 2}(s)$, our result is more explicit than theirs. See [13, Theorems $5-7]$ for their results.

We mention an underlying idea of the proof of Theorem 1.1. In [7] Deninger used an improvement of Weil's explicit formula due to Barner [5] to obtain Deninger's Theorem 1.1. But, at present, we cannot use a Weil's-type explicit formula [13, Theorem 4] for our purpose. Instead, we adopt the contour integral method, which was used for appropriate sums of multiple Hurwitz zeta functions or for multiple sine functions in $[4,16]$. See also [19, Section 2.4] for this method. Roughly speaking, we give an analytic continuation with respect to $w$ for

$$
\sum_{\operatorname{Im}\left(\rho_{1}\right)>0} \sum_{\operatorname{Im}\left(\rho_{2}\right)>0}\left(s-\rho_{1}-\rho_{2}\right)^{-w}
$$

via the contour integral expression and cut off the contour to relate (1.2) with prime numbers. Then the theory of Cramér [6] and Guinand [8], which will be explained in Section 2, plays important roles. More technical ideas will be explained in Section 3. We emphasize that Theorem 3.1 in Section 3 is fundamental to derive Theorem 1.1. We remark that for concretely given $r \in \mathbb{Z}_{\geq 1}$ Deninger's-type explicit formulas for $\zeta^{\otimes r}(s)$ can be calculated from Theorem 3.1 in principle.

This paper is organized as follows: In Section 2 we recall the theory of Cramér [6] and Guinand [8], arrange it and give needed estimations. In Section 3 we show a fundamental equation (Theorem 3.1) to obtain Theorem 1.1 and we also explain a sketch of the proof of Theorem 1.1. In Section 4 we reprove Deninger's Theorems 1.1 and 1.2 from Theorem 3.1 to explain calculations for Theorems 1.1 and 1.3. We remark that our method is completely different from that of Deninger. In Section 5 we prove Theorem 1.1 from Theorem 3.1 and we also prove Theorem 1.2. In Section 6 we prove 
Theorem 1.3. Lastly, in Section 7 we discuss an obstacle to applying our results to the nontrivial zeros of the Riemann zeta function.

Convention and notation. For convenience we collect convention and notation which will be used in this paper.

- $p$ and $q$ denote the prime numbers.

- $\rho, \rho_{1}$ and $\rho_{2}$ denote the nontrivial zeros of the Riemann zeta function counted with multiplicity.

- $\tau$ denotes the complex number satisfying $\rho=\frac{1}{2}+\mathrm{i} \tau$.

- $\tau^{(1)}:=\min \left\{\operatorname{Re}(\tau)>0: \zeta\left(\frac{1}{2}+\mathrm{i} \tau\right)=0\right\}(=14.13 \ldots)$. See [19, Chapter $\mathrm{XV}]$.

- $\gamma$ is the Euler constant, that is,

$$
\gamma:=\lim _{N \rightarrow \infty}\left(\sum_{n=1}^{N} \frac{1}{n}-\log N\right) .
$$

\section{Cramér-Guinand theory}

In this section, we recall the theory of Cramér [6] and Guinand [8] and further develop it. Let

$$
\theta(t):=\sum_{\operatorname{Re}(\tau)>0} \mathrm{e}^{-\tau t}, \quad U(t):=\theta(t)+\frac{\log t}{4 \pi \sin (t / 2)},
$$

which are originally defined in $|\arg (t)|<\pi / 2$. Then, Cramér and Guinand proved the following assertions:

Lemma 2.1. (1) $[6$, p. 114, (13)] $\theta(t)$ has the following expression in $\operatorname{Re}(t)>0$ :

$$
\begin{aligned}
\theta(t)= & \frac{t}{2 \pi \mathrm{i}} \mathrm{e}^{\mathrm{i} t} \sum_{p} \sum_{m=1}^{\infty} \frac{p^{-m}}{m(t+\mathrm{i} m \log p)}-\frac{t}{2 \pi \mathrm{i}} \mathrm{e}^{-\frac{\mathrm{i} t}{2}} \sum_{p} \sum_{m=1}^{\infty} \frac{p^{-m}}{m(t-\mathrm{i} m \log p)} \\
& +\mathrm{e}^{-\frac{\mathrm{i} t}{2}}\left(\frac{1}{4}+\frac{\gamma+\log (2 \pi)}{2 \pi \mathrm{i}}\right)\left(1+\frac{1}{\mathrm{i} t}\right)+\frac{\mathrm{e}^{-\frac{\mathrm{i} t}{2}}}{2 \pi \mathrm{i}} \frac{\Gamma^{\prime}}{\Gamma}\left(\frac{t}{\pi}\right)+\frac{\mathrm{e}^{\frac{\mathrm{i} t}{2}}}{2} \\
& -\frac{t}{2 \pi} \mathrm{e}^{-\frac{\mathrm{i} t}{2}} \int_{0}^{1} \mathrm{e}^{\mathrm{i} t u} \log |\zeta(u)| d u+\frac{\mathrm{e}^{-\frac{\mathrm{i} t}{2}}}{2 \pi t} \int_{0}^{\infty} \frac{u}{\mathrm{e}^{u}-1} \frac{d u}{u+\mathrm{i} t} .
\end{aligned}
$$


(2) [8, Theorem 3] $U(t)$ has a single valued meromorphic continuation for which

$$
U(t)+U(-t)=2 \cos \left(\frac{t}{2}\right)-\frac{1}{4 \cos (t / 2)} .
$$

(3) $[6$, p. 117, 2; 8, Theorem 1(ii)] $U(t)$ has the following Laurent expansion at $t=0$ :

$$
U(t)=-\frac{\gamma+\log (2 \pi)}{2 \pi t}+O(1)
$$

Remark 2.1. The sums over $p$ and $m$ in Lemma 2.1(1) converge absolutely and uniformly on any compact subset of $\mathbb{C}$ since

$$
\sum_{p} \sum_{m=1}^{\infty} \frac{p^{-m}}{m^{2} \log p}<\infty .
$$

For the proof of (2.2), we separate $m=1$ and $m \geq 2$ and apply the prime number theorem to the former and estimate the latter trivially.

From the meromorphy of $\log t /(4 \pi \sin (t / 2))$ in $\arg (t) \in(-\pi / 2,(3 \pi) / 2)$ and Lemma 2.1(2), $\theta(t)$ has a meromorphic continuation to $t \in \mathbb{C} \backslash i \mathbb{R}_{\leq 0}$. We rewrite it by the same notation $\theta(t) .{ }^{1}$ We put

$$
\theta^{*}(t):=\theta(t)-\mathrm{e}^{-\frac{\mathrm{i} t}{2}} \quad\left(t \in \mathbb{C} \backslash \mathrm{i} \mathbb{R}_{\leq 0}\right)
$$

Then we rewrite Lemma 2.1 in terms of $\theta^{*}(t)$ as follows:

Lemma 2.2. (1) $\theta^{*}(t)$ has the following expression in $t \in \mathbb{C} \backslash i \mathbb{R}_{\leq 0}$ :

$$
\theta^{*}(t)=\sum_{j=1}^{8} A_{j}(t)
$$

where

$$
A_{1}(t):=-\frac{t}{2 \pi \mathrm{i}} \mathrm{e}^{-\frac{\mathrm{i} t}{2}} \sum_{p} \sum_{m=1}^{\infty} \frac{p^{-m}}{m(t-\mathrm{i} m \log p)}
$$

\footnotetext{
${ }^{1}$ Throughout this paper $\theta$ is a function in $\mathbb{C} \backslash i \mathbb{R}_{\leq 0}$ and we do not use other branches.
} 


$$
\begin{aligned}
& A_{2}(t):=\frac{t}{2 \pi \mathrm{i}} \mathrm{e}^{\frac{\mathrm{i} t}{2}} \sum_{p} \sum_{m=1}^{\infty} \frac{p^{-m}}{m(t+\mathrm{i} m \log p)}, \\
& A_{3}(t):=-\mathrm{e}^{\frac{\mathrm{i} t}{2}}\left(\frac{1}{4}+\frac{\gamma+\log (2 \pi)}{2 \pi \mathrm{i}}\right)\left(1-\frac{1}{\mathrm{i} t}\right), \\
& A_{4}(t):=-\frac{\mathrm{e}^{\frac{\mathrm{i} t}{2}}}{2 \pi \mathrm{i}} \frac{\Gamma^{\prime}}{\Gamma}\left(-\frac{t}{\pi}\right), \\
& A_{5}(t):=-\frac{t}{2 \pi} \mathrm{e}^{\frac{\mathrm{i} t}{2}} \int_{0}^{1} \mathrm{e}^{-\mathrm{i} t u} \log |\zeta(u)| d u, \\
& A_{6}(t):=\frac{\mathrm{e}^{\frac{\mathrm{i} t}{2}}}{2 \pi t} \int_{0}^{\infty} \frac{u}{\mathrm{e}^{u}-1} \frac{d u}{u-\mathrm{i} t}, \\
& A_{7}(t):=-\frac{1}{2} \mathrm{e}^{-\frac{\mathrm{i} t}{2}}, \quad A_{8}(t):=\frac{\mathrm{e}^{\frac{3 \mathrm{i} t}{2}}}{\mathrm{e}^{\mathrm{i} t}-\mathrm{e}^{-\mathrm{i} t} .}
\end{aligned}
$$

(2) We have

$$
\theta^{*}(t)+\theta^{*}(-t)= \begin{cases}-\frac{\mathrm{e}^{\frac{\mathrm{i} t}{2}}}{2 \mathrm{i} \sin t} & \text { if } \operatorname{Re}(t)>0 \\ \frac{\mathrm{e}^{-\frac{\mathrm{i} t}{2}}}{2 \mathrm{i} \sin t} & \text { if } \operatorname{Re}(t)<0 .\end{cases}
$$

(3) $\theta^{*}(t)$ has the following asymptotic behavior at $t=0$ :

$$
\theta^{*}(t)=-\frac{\log t}{2 \pi t}-\frac{\gamma+\log (2 \pi)}{2 \pi t}+O(1)
$$

where the argument lies in $(-\pi / 2,(3 \pi) / 2)$.

Remark 2.2. For the proof of (1) we cannot use Lemma 2.1(1) directly because the last term in Lemma 2.1(1) is not valid on $t \in \mathrm{i} \mathbb{R}_{\geq 0}$.

Proof of Lemma 2.2. (2) First we consider the case $\operatorname{Re}(t)>0$. We recall that the argument was taken in $(-\pi / 2,(3 \pi) / 2)$ to obtain the meromorphic continuation of $\theta(t)$. Hence $\log (-t)=\log t+\pi \mathrm{i}$ in its context. Therefore Lemma 2.1(2) says

$$
\theta(t)+\theta(-t)+\frac{1}{4 \mathrm{i} \sin (t / 2)}=2 \cos \left(\frac{t}{2}\right)-\frac{1}{4 \cos (t / 2)} .
$$


Hence we have

$$
\begin{aligned}
\theta^{*}(t)+\theta^{*}(-t) & =\theta(t)+\theta(-t)-2 \cos \left(\frac{t}{2}\right) \\
& =-\frac{1}{4 \cos (t / 2)}-\frac{1}{4 \mathrm{i} \sin (t / 2)}=-\frac{\mathrm{e}^{\frac{\mathrm{i} t}{2}}}{2 \mathrm{i} \sin t}
\end{aligned}
$$

which gives (2) with $\operatorname{Re}(t)>0$. (2) with $\operatorname{Re}(t)<0$ is immediately obtained by replacing $t$ with $-t$ in $(2)$ with $\operatorname{Re}(t)>0$.

(1) We restrict $t$ to $\operatorname{Re}(t)<0$ first. Then from (2) we have

$$
\theta^{*}(t)=-\theta(-t)+\mathrm{e}^{\frac{\mathrm{i} t}{2}}+\frac{\mathrm{e}^{-\frac{\mathrm{i} t}{2}}}{2 \mathrm{i} \sin t}=-\theta(-t)+\frac{\mathrm{e}^{\frac{3 i t}{2}}}{\mathrm{e}^{\mathrm{i} t}-\mathrm{e}^{-\mathrm{i} t}} .
$$

Applying Lemma 2.1(1) to $\theta(-t)$, we obtain (1) in $\operatorname{Re}(t)<0$. Since both sides are meromorphic in $t \in \mathbb{C} \backslash \mathrm{i} \mathbb{R}_{\leq 0}$, (1) holds in $t \in \mathbb{C} \backslash \mathrm{i} \mathbb{R}_{\leq 0}$.

(3) Applying $\sin (t / 2)=t / 2+O\left(t^{3}\right)$ to Lemma 2.1(3), we immediately obtain the desired result.

Next, we give information about poles of $\theta^{*}(t)$. From definition (2.1) of $\theta(t)$ and Lemma $2.2(1)$ we have

Lemma 2.3 [8, Theorem $1(\mathrm{ii})] . \theta^{*}(t)$ has poles at the following points and nowhere else in $t \in \mathbb{C} \backslash \mathrm{i} \mathbb{R}_{\leq 0}$ :

(i) $t=\mathrm{i} m \log p(p:$ prime numbers; $m=1,2, \ldots)$,

(ii) $t=-\pi m(m=1,2, \ldots)$.

Later we need the following bounds for $\theta^{*}(t)$ :

Lemma 2.4. (1) On $\operatorname{Re}(t) \geq 1$ we have

$$
\theta^{*}(t)=\sum_{0<\tau \leq 100} \mathrm{e}^{-\tau t}-\mathrm{e}^{-\frac{\mathrm{i} t}{2}}+O\left(\mathrm{e}^{-100 \operatorname{Re}(t)+\frac{1}{2}|\operatorname{Im}(t)|}\right)
$$

(2) On $\operatorname{Re}(t) \leq-1$ we have

$$
\theta^{*}(t)=-\sum_{0<\tau \leq 100} \mathrm{e}^{\tau t}+\frac{\mathrm{e}^{\frac{3 \mathrm{i} t}{2}}}{\mathrm{e}^{\mathrm{i} t}-\mathrm{e}^{-\mathrm{i} t}}+O\left(\mathrm{e}^{100 \operatorname{Re}(t)+\frac{1}{2}|\operatorname{Im}(t)|}\right)
$$


(3) Let $t=x+\mathrm{i} T$ with $T \geq 2$ and $-T \leq x \leq T$. Then we have

$$
\theta^{*}(t)=-\frac{t}{2 \pi \mathrm{i}} \mathrm{e}^{-\frac{\mathrm{i} t}{2}} \sum_{\substack{p, m \\ p^{m}<\mathrm{e}^{2 T}}} \frac{p^{-m}}{m(t-\mathrm{i} m \log p)}+O\left(T \mathrm{e}^{T / 2}\right)
$$

where the implied constant is absolute.

Proof. (1) First we remark that if $0<\operatorname{Re}(\tau) \leq 100$ and $\zeta\left(\frac{1}{2}+\mathrm{i} \tau\right)=0$, then $\tau \in \mathbb{R}$ (see [19, Chapter XV]). Hence we have

$$
\theta^{*}(t)=\sum_{0<\tau \leq 100} \mathrm{e}^{-\tau t}+\sum_{\operatorname{Re}(\tau)>100} \mathrm{e}^{-\tau t}-\mathrm{e}^{-\frac{\mathrm{i} t}{2}} .
$$

From $|\operatorname{Im}(\tau)|<\frac{1}{2}$ we have

$$
\begin{aligned}
\left|\sum_{\operatorname{Re}(\tau)>100} \mathrm{e}^{-\tau t}\right| & \leq \sum_{\operatorname{Re}(\tau)>100}\left|\mathrm{e}^{-\tau t}\right|=\sum_{\operatorname{Re}(\tau)>100} \mathrm{e}^{-\operatorname{Re}(\tau) \operatorname{Re}(t)+\operatorname{Im}(\tau) \operatorname{Im}(t)} \\
& \leq \mathrm{e}^{\frac{1}{2}|\operatorname{Im}(t)|} \sum_{\operatorname{Re}(\tau)>100} \mathrm{e}^{-\operatorname{Re}(\tau) \operatorname{Re}(t)} \\
& =\mathrm{e}^{-100 \operatorname{Re}(t)+\frac{1}{2}|\operatorname{Im}(t)|} \sum_{\operatorname{Re}(\tau)>100} \mathrm{e}^{-(\operatorname{Re}(\tau)-100) \operatorname{Re}(t)} \\
& \leq \mathrm{e}^{-100 \operatorname{Re}(t)+\frac{1}{2}|\operatorname{Im}(t)|} \sum_{\operatorname{Re}(\tau)>100} \mathrm{e}^{-(\operatorname{Re}(\tau)-100)}
\end{aligned}
$$

which completes the proof.

(2) Applying (1) to $\theta^{*}(-t)$ in Lemma 2.2(2), we easily obtain the desired result.

(3) Estimating $A_{j}(t)$ in Lemma $2.2(1)$ for $j=2,3, \ldots, 8$ trivially, we have

$$
\theta^{*}(t)=A_{1}(t)+O\left(T \mathrm{e}^{T / 2}\right)
$$

We treat $A_{1}(t)$. Suppose that $p^{m} \geq \mathrm{e}^{2 T}$. Then, since $T \leq(m \log p) / 2$, we have $m \log p-T \geq(m \log p) / 2$. Hence we get

$$
\left|\sum_{\substack{p, m \\ p^{m} \geq \mathrm{e}^{2 T}}} \frac{p^{-m}}{m(t-\mathrm{i} m \log p)}\right| \leq \sum_{\substack{p, m \\ p^{m} \geq \mathrm{e}^{2 T}}} \frac{p^{-m}}{m(m \log p-T)} \leq 2 \sum_{\substack{p, m \\ p^{m} \geq \mathrm{e}^{2 T}}} \frac{p^{-m}}{m^{2} \log p}
$$




$$
\leq 2 \sum_{p} \sum_{m=1}^{\infty} \frac{p^{-m}}{m^{2} \log p}
$$

Together with (2.2), this completes the proof.

Corollary 2.1. (1) On $u \geq 10$ we have

$$
\theta^{*}\left(u \mathrm{e}^{-\frac{\pi \mathrm{i}}{4}}\right)=O\left(\exp \left(-\frac{u}{2^{3 / 2}}\right)\right), \quad \theta^{*}\left(u \mathrm{e}^{\frac{3 \pi \mathrm{i}}{4}}\right)=O\left(\exp \left(-\frac{5 u}{2^{3 / 2}}\right)\right)
$$

(2) Let $R \geq 10$ and $-R \leq y \leq R$. Then we have

$$
\theta^{*}(R+\mathrm{i} y)=O\left(\mathrm{e}^{y / 2}\right)
$$

where the implied constant is absolute.

(3) Let $t=x+\mathrm{i} T$ with $T=\log \left(N+\frac{1}{2}\right)\left(N \in \mathbb{Z}_{\geq 100}\right)$ and $x \in \mathbb{R}$. Then we have

$$
\theta^{*}(t)= \begin{cases}O\left(\mathrm{e}^{T / 2}\right) & \text { if } x \geq 1 \\ O\left(T^{2} \mathrm{e}^{T / 2}\right) & \text { if }-1 \leq x \leq 1 \\ O\left(\mathrm{e}^{\tau^{(1)} x}+\mathrm{e}^{-\frac{5}{2} T}+\mathrm{e}^{100 x+\frac{T}{2}}\right) & \text { if } x \leq-1\end{cases}
$$

where the implied constant is absolute.

For the proof we need the following:

Lemma 2.5. For any $0<X<Y$ we have

$$
\log Y-\log X \geq \frac{Y-X}{Y}
$$

Proof. This immediately follows from the mean value theorem.

Proof of Corollary 2.1. Applying Lemma 2.4(1) or (2) and estimating the first and second terms trivially, we easily obtain (1) to (3) with $|x| \geq 1$. We 
consider (3) with $|x| \leq 1$. We estimate the sum in Lemma 2.4(3):

$$
\begin{aligned}
& \left|\sum_{\substack{p, m \\
p^{m}<\mathrm{e}^{2 T}}} \frac{p^{-m}}{m(t-\mathrm{i} m \log p)}\right| \leq \sum_{\substack{p, m \\
p^{m}<\mathrm{e}^{2 T}}} \frac{p^{-m}}{|T-m \log p|} \\
& \quad=\sum_{\substack{p, m \\
p^{m}<N+\frac{1}{2}}} \frac{p^{-m}}{T-m \log p}+\sum_{\substack{p, m \\
N+\frac{1}{2} \leq p^{m}<\left(N+\frac{1}{2}\right)^{2}}} \frac{p^{-m}}{m \log p-T} \\
& = \\
& =\mathrm{I}+\mathrm{II} .
\end{aligned}
$$

We first treat I. From Lemma 2.5 we have

$$
\begin{aligned}
\mathrm{I} & \leq\left(N+\frac{1}{2}\right) \sum_{\substack{p, m \\
p^{m}<N+\frac{1}{2}}} \frac{p^{-m}}{N+\frac{1}{2}-p^{m}} \leq\left(N+\frac{1}{2}\right) \sum_{n=2}^{N} \frac{1}{n\left(N+\frac{1}{2}-n\right)} \\
& =\sum_{n=2}^{N} \frac{1}{n}+\sum_{n=2}^{N} \frac{1}{N+\frac{1}{2}-n} \ll \log N \ll T .
\end{aligned}
$$

In the same manner, II is estimated as follows:

$$
\mathrm{II} \leq \sum_{\substack{p, m \\ N+\frac{1}{2} \leq p^{m}<\left(N+\frac{1}{2}\right)^{2}}} \frac{1}{p^{m}-\left(N+\frac{1}{2}\right)} \leq \sum_{n=1}^{N^{2}} \frac{1}{(n+N)-\left(N+\frac{1}{2}\right)} \ll T .
$$

Applying (2.3) and (2.4) to Lemma 2.4(3), we get the desired result.

\section{The fundamental equation between zeros and primes}

In this section, we prove a fundamental equation between the zeros of the Riemann zeta function and the prime numbers. We also explain how to obtain Deninger's Theorem 1.1 and Theorem 1.1 from the fundamental equation. Needed calculations will be done in Sections 4 and 5 .

The fundamental equation is as follows: 
Theorem 3.1. Let $r \in \mathbb{Z}_{\geq 1}$. Suppose that $(w, z) \in D_{r}$ satisfies $\operatorname{Im}(z)<$ $-r / 2$ and $\operatorname{Re}(w)>r$, where

$$
\begin{aligned}
D_{r} & :=\left\{(w, z) \in \mathbb{C}^{2}:-\frac{r}{2^{3 / 2}}<\operatorname{Re}\left(z \mathrm{e}^{-\frac{\pi \mathrm{i}}{4}}\right)<\frac{5 r}{2^{3 / 2}}\right\} \\
& =\left\{(w, z) \in \mathbb{C}^{2}:-\frac{r}{2}<\operatorname{Re}(z)+\operatorname{Im}(z)<\frac{5 r}{2}\right\} .
\end{aligned}
$$

Then we have

$$
L_{r}^{(1)}(w, z)+L_{r}^{(2)}(w, z)=R_{r}(w, z)
$$

where

$$
\begin{aligned}
L_{r}^{(1)}(w, z) & :=\frac{1}{\Gamma(w)} \int_{0}^{\infty \mathrm{e}^{-\frac{\pi \mathrm{i}}{4}}} \mathrm{e}^{-z t} \theta^{*}(t)^{r} t^{w-1} d t \\
L_{r}^{(2)}(w, z) & :=(-1)^{r-1} \frac{\mathrm{e}^{\pi \mathrm{i} w}}{\Gamma(w)} \int_{0}^{\infty \mathrm{e}^{-\frac{\pi \mathrm{i}}{4}}} \mathrm{e}^{z t}\left(\theta(t)+\sum_{n=1}^{\infty} \mathrm{e}^{-\left(2 n+\frac{1}{2}\right) \mathrm{i} t}\right)^{r} t^{w-1} d t \\
R_{r}(w, z) & :=\frac{2 \pi \mathrm{i}}{\Gamma(w)} \lim _{N \rightarrow \infty} \sum_{\substack{p, m \\
p^{m}<N+\frac{1}{2}}} \operatorname{Res}_{t=\mathrm{i} m \log p} \mathrm{e}^{-z t} \theta^{*}(t)^{r} t^{w-1}
\end{aligned}
$$

and the argument lies in $(-\pi / 2,(3 \pi) / 2)$.

Before we start the proof, we explain how to get Deninger's Theorem 1.1 and Theorem 1.1 from Theorem 3.1. Let $r=1$ or $r=2$. We calculate the residue in $R_{r}(w, z)$ explicitly by Lemma $2.2(1)$. On the other hand, we calculate $L_{r}^{(1)}(w, z)$ and $L_{r}^{(2)}(w, z)$ by definition $(2.1)$ of $\theta(t)$ and the following formula for the gamma function:

Lemma 3.1. Let $\arg \alpha \in(-\pi / 4,(3 \pi) / 4)$ and $\operatorname{Re}(w)>0$. Then we have

$$
\frac{\Gamma(w)}{\alpha^{w}}=\int_{0}^{\infty \mathrm{e}^{-\frac{\pi \mathrm{i}}{4}}} \mathrm{e}^{-\alpha t} t^{w-1} d t
$$

Proof. First we restrict $\alpha$ to $\arg \alpha=\pi / 4$. Then we have

$$
\frac{\Gamma(w)}{\alpha^{w}}=\int_{0}^{\infty} \mathrm{e}^{-t}\left(\frac{t}{\alpha}\right)^{w} \frac{d t}{t}=\int_{0}^{\infty \alpha^{-1}} \mathrm{e}^{-\alpha t} t^{w} \frac{d t}{t}=\int_{0}^{\infty \mathrm{e}^{-\frac{\pi \mathrm{i}}{4}}} \mathrm{e}^{-\alpha t} t^{w} \frac{d t}{t}
$$

When $w$ is fixed, both sides are holomorphic in $\left\{\alpha \in \mathbb{C}: \operatorname{Re}\left(\alpha \mathrm{e}^{-\frac{\pi \mathrm{i}}{4}}\right)>0\right\}=$ $\{\alpha \in \mathbb{C}:-\pi / 4<\arg \alpha<(3 \pi) / 4\}$. This completes the proof. 
Finally replacing $z=-\mathrm{i}\left(s-\frac{r}{2}\right)$ and investigating holomorphy of both sides with respect to $s$, we will obtain Deninger's Theorem 1.1 and Theorem 1.1. (Here the assumptions of Theorem 3.1 concerning $(w, z)$ is equivalent to $-2 r<\operatorname{Re}(s)-\operatorname{Im}(s)<r, \operatorname{Re}(s)>r$ and $\operatorname{Re}(w)>r$. $)$

Proof of Theorem 3.1. For a fixed number $0<\varepsilon<\log 2$ we consider

$$
F_{r}(w, z)=F_{r}^{(\varepsilon)}(w, z):=\frac{1}{\Gamma(w)} \int_{C_{\varepsilon}} \mathrm{e}^{-z t} \theta^{*}(t)^{r} t^{w-1} d t,
$$

where $C_{\varepsilon}$ is the union of $\infty \mathrm{e}^{\frac{3 \pi}{4} \mathrm{i}} \rightarrow \varepsilon \mathrm{e}^{\frac{3 \pi}{4}}, \varepsilon \mathrm{e}^{\mathrm{i} \phi}\left(\phi: \frac{3 \pi}{4} \rightarrow-\frac{\pi}{4}\right)$, and $\varepsilon \mathrm{e}^{-\frac{\pi \mathrm{i}}{4}} \rightarrow$ $\infty \mathrm{e}^{-\frac{\pi \mathrm{i}}{4}} .{ }^{2}$ Owing to Corollary 2.1(1), (3.1) converges absolutely and uniformly on any compact subset of $D_{r}$. Let $(w, z) \in D_{r}$ and $0<\delta<\varepsilon$. Then from Cauchy's theorem and Lemma 2.3 we have

$$
F_{r}(w, z)-F_{r}^{(\delta)}(w, z)=\frac{1}{\Gamma(w)} \int_{P_{\delta, \varepsilon}} \mathrm{e}^{-z t} \theta^{*}(t)^{r} t^{w-1} d t=0,
$$

where $P_{\delta, \varepsilon}$ is the union of $\varepsilon \mathrm{e}^{\mathrm{i} \phi}\left(\phi: \frac{3 \pi}{4} \rightarrow-\frac{\pi}{4}\right), \varepsilon \mathrm{e}^{-\frac{\pi \mathrm{i}}{4}} \rightarrow \delta \mathrm{e}^{-\frac{\pi \mathrm{i}}{4}}, \delta \mathrm{e}^{\mathrm{i} \phi}\left(\phi:-\frac{\pi}{4} \rightarrow\right.$ $\left.\frac{3 \pi}{4}\right)$ and $\delta \mathrm{e}^{\frac{3 \pi}{4} \mathrm{i}} \rightarrow \varepsilon \mathrm{e}^{\frac{3 \pi}{4} \mathrm{i}}$. We restrict $w$ to $\operatorname{Re}(w)>r$. Taking the limit $\delta \downarrow 0$, from Lemma 2.2(3), we get

$$
\begin{aligned}
& F_{r}(w, z)=\lim _{\delta \downarrow 0} F_{r}^{(\delta)}(w, z) \\
& (3.2) \quad=\frac{1}{\Gamma(w)} \int_{\infty \mathrm{e}^{\frac{3 \pi \mathrm{i}}{4}}}^{0} \mathrm{e}^{-z t} \theta^{*}(t)^{r} t^{w-1} d t+\frac{1}{\Gamma(w)} \int_{0}^{\infty \mathrm{e}^{-\frac{\pi \mathrm{i}}{4}}} \mathrm{e}^{-z t} \theta^{*}(t)^{r} t^{w-1} d t .
\end{aligned}
$$

Replacing $t$ with $-t$ and applying Lemma 2.2(2), we calculate the first term of (3.2) as follows:

$$
\begin{aligned}
& \frac{1}{\Gamma(w)} \int_{\infty \mathrm{e}^{\frac{3 \pi}{4} \mathrm{i}}}^{0} \mathrm{e}^{-z t} \theta^{*}(t)^{r} t^{w-1} d t \\
& \quad=\frac{1}{\Gamma(w)} \int_{0}^{\infty \mathrm{e}^{-\frac{\pi \mathrm{i}}{4}}} \mathrm{e}^{z t} \theta^{*}(-t)^{r} \mathrm{e}^{\pi \mathrm{i}(w-1)} t^{w-1} d t \\
& \quad=(-1)^{r-1} \frac{\mathrm{e}^{\pi \mathrm{i} w}}{\Gamma(w)} \int_{0}^{\infty \mathrm{e}^{-\frac{\pi \mathrm{i}}{4}}} \mathrm{e}^{z t}\left(\theta^{*}(t)+\frac{\mathrm{e}^{\frac{\mathrm{i} t}{2}}}{2 \mathrm{i} \sin t}\right)^{r} t^{w-1} d t .
\end{aligned}
$$

\footnotetext{
${ }^{2}$ About the contour $C_{\varepsilon}$ we chose the angle $-\pi / 4$ and $(3 \pi) / 4$ so as not to exist poles of $\theta^{*}(t)$ on $C_{\varepsilon}$. The choice of the angle is not essential.
} 
Together with $-\mathrm{e}^{-\frac{\mathrm{i} t}{2}}+\frac{\mathrm{e}^{\mathrm{it}} \frac{\mathrm{t}}{2 \mathrm{i} \sin t}}{2}=-\mathrm{e}^{-\frac{\mathrm{i} t}{2}}+\frac{\mathrm{e}^{-\frac{\mathrm{it}}{2}}}{1-\mathrm{e}^{-2 \mathrm{it}}}=\sum_{n=1}^{\infty} \mathrm{e}^{-\left(2 n+\frac{1}{2}\right) \mathrm{it}}$, turns to

$$
F_{r}(w, z)=L_{r}^{(1)}(w, z)+L_{r}^{(2)}(w, z) \quad\left((w, z) \in D_{r}, \operatorname{Re}(w)>r\right) .
$$

We calculate $F_{r}(w, z)$ by the residue theorem. Let $(w, z) \in D_{r}$ satisfy $\operatorname{Im}(z)<$ $-r / 2$ and be fixed. Then from the residue theorem and Lemma 2.3 we have

$$
\begin{aligned}
& \left(\int_{C^{(1)}}+\int_{C^{(2)}}+\int_{C^{(3)}}\right) \mathrm{e}^{-z t} \theta^{*}(t)^{r} t^{w-1} d t \\
& \quad=2 \pi \mathrm{i} \sum_{\substack{p, m \\
p^{m}<N+\frac{1}{2}}} \operatorname{Res}_{t=\mathrm{i} m \log p} \mathrm{e}^{-z t} \theta^{*}(t)^{r} t^{w-1},
\end{aligned}
$$

where

$$
\begin{aligned}
C^{(1)}:= & \left\{-u+\mathrm{i} u \mid u: T \rightarrow \frac{\varepsilon}{\sqrt{2}}\right\} \cup\left\{\varepsilon \mathrm{e}^{\mathrm{i} \phi} \mid \phi: \frac{3 \pi}{4} \rightarrow-\frac{\pi}{4}\right\} \\
& \cup\left\{u-\mathrm{i} u \mid u: \frac{\varepsilon}{\sqrt{2}} \rightarrow R\right\}, \\
C^{(2)}:= & \{R+\mathrm{i} y \mid y:-R \rightarrow T\}, \quad C^{(3)}:=\{x+\mathrm{i} T \mid x: R \rightarrow-T\},
\end{aligned}
$$

$T=T_{N}:=\log \left(N+\frac{1}{2}\right)$ with $N \in \mathbb{Z}_{\geq 100}$ and $R \geq T$. We consider the limit $R \rightarrow \infty$. From Corollary 2.1(2) we have

$$
\begin{aligned}
\left|\int_{C^{(2)}} \mathrm{e}^{-z t} \theta^{*}(t)^{r} t^{w-1} d t\right| & =\left|\int_{-R}^{T} \mathrm{e}^{-z(R+\mathrm{i} y)} \theta^{*}(R+\mathrm{i} y)^{r}(R+\mathrm{i} y)^{w-1} \mathrm{i} d y\right| \\
& \ll_{r, w} \int_{-R}^{T} \mathrm{e}^{-\operatorname{Re}(z) R+\operatorname{Im}(z) y} \mathrm{e}^{\frac{r}{2} y} R^{\operatorname{Re}(w)-1} d y \\
& \leq 2 R^{\operatorname{Re}(w)} \mathrm{e}^{-\left(\operatorname{Re}(z)+\operatorname{Im}(z)+\frac{r}{2}\right) R} .
\end{aligned}
$$

Here in the final inequality we used $T \leq R$ and $\operatorname{Im}(z)<-r / 2$. From $\operatorname{Re}(z)+$ $\operatorname{Im}(z)+\frac{r}{2}>0,(3.4)$ tends to zero as $R \rightarrow \infty$. Hence we get

$$
\left(\int_{C^{(4)}}+\int_{C^{(5)}}\right) \mathrm{e}^{-z t} \theta^{*}(t)^{r} t^{w-1} d t=2 \pi \mathrm{i} \sum_{\substack{p, m \\ p^{m}<N+\frac{1}{2}}} \operatorname{Res}_{t=\mathrm{i} m \log p} \mathrm{e}^{-z t} \theta^{*}(t)^{r} t^{w-1},
$$


where

$$
\begin{aligned}
C^{(4)}:= & \left\{-u+\mathrm{i} u \mid u: T \rightarrow \frac{\varepsilon}{\sqrt{2}}\right\} \cup\left\{\varepsilon \mathrm{e}^{\mathrm{i} \phi} \mid \phi: \frac{3 \pi}{4} \rightarrow-\frac{\pi}{4}\right\} \\
& \cup\left\{u-\mathrm{i} u \mid u: \frac{\varepsilon}{\sqrt{2}} \rightarrow \infty\right\}, \\
C^{(5)}:= & \{x+\mathrm{i} T \mid x: \infty \rightarrow-T\} .
\end{aligned}
$$

Now we consider the limit $N \rightarrow \infty$. We have

$$
\begin{aligned}
\left|\int_{C^{(5)}}\right| & =\left|-\int_{-T}^{\infty} \mathrm{e}^{-z(x+\mathrm{i} T)} \theta^{*}(x+\mathrm{i} T)^{r}(x+\mathrm{i} T)^{w-1} d x\right| \\
& \ll w \int_{-T}^{\infty} \mathrm{e}^{-\operatorname{Re}(z) x+\operatorname{Im}(z) T}\left|\theta^{*}(x+\mathrm{i} T)\right|^{r} \max \{|x|, T\}^{\operatorname{Re}(w)-1} d x \\
& =\int_{-T}^{-1}+\int_{-1}^{1}+\int_{1}^{\infty}
\end{aligned}
$$

We deal with $\int_{1}^{\infty}$ in (3.5). From Corollary 2.1(3) we have

$$
\begin{aligned}
\int_{1}^{\infty} & \ll_{r} \int_{1}^{\infty} \mathrm{e}^{-\operatorname{Re}(z) x+\operatorname{Im}(z) T} \mathrm{e}^{\frac{r}{2} T} \max \{x, T\}^{\operatorname{Re}(w)-1} d x \\
& =\mathrm{e}^{\left(\operatorname{Im}(z)+\frac{r}{2}\right) T}\left(T^{\operatorname{Re}(w)-1} \int_{1}^{T} \mathrm{e}^{-\operatorname{Re}(z) x} d x+\int_{T}^{\infty} \mathrm{e}^{-\operatorname{Re}(z) x} x^{\operatorname{Re}(w)-1} d x\right) \\
& \ll_{w, z} \mathrm{e}^{\left(\operatorname{Im}(z)+\frac{r}{2}\right) T}\left(T^{\operatorname{Re}(w)-1}+1\right) .
\end{aligned}
$$

Here in the final inequality we used $\operatorname{Re}(z)>-\left(\operatorname{Im}(z)+\frac{r}{2}\right)>0$. From $\operatorname{Im}(z)+\frac{r}{2}<0$ we get $\int_{1}^{\infty} \rightarrow 0$ as $N \rightarrow \infty$. Next, we consider $\int_{-1}^{1}$ in (3.5). From Corollary 2.1(3) we have

$$
\begin{aligned}
\int_{-1}^{1} & \ll_{r} \int_{-1}^{1} \mathrm{e}^{-\operatorname{Re}(z) x+\operatorname{Im}(z) T}\left(T^{2} \mathrm{e}^{T / 2}\right)^{r} T^{\operatorname{Re}(w)-1} d x \\
& \ll_{z} T^{\operatorname{Re}(w)+2 r-1} \mathrm{e}^{\left(\operatorname{Im}(z)+\frac{r}{2}\right) T} \rightarrow 0 \quad \text { as } N \rightarrow \infty
\end{aligned}
$$


Finally we treat $\int_{-T}^{-1}$ in $(3.5)$. From Corollary $2.1(3)$ we have

$$
\begin{aligned}
\int_{-T}^{-1} \ll & \ll_{-T}^{-1} \mathrm{e}^{-\operatorname{Re}(z) x+\operatorname{Im}(z) T}\left(\mathrm{e}^{\tau^{(1)} r x}+\mathrm{e}^{-\frac{5}{2} r T}+\mathrm{e}^{100 r x+\frac{r}{2} T}\right) T^{\operatorname{Re}(w)-1} d x \\
= & T^{\operatorname{Re}(w)-1} \mathrm{e}^{\operatorname{Im}(z) T} \int_{-T}^{-1} \mathrm{e}^{\left(\tau^{(1)} r-\operatorname{Re}(z)\right) x} d x \\
& +T^{\operatorname{Re}(w)-1} \mathrm{e}^{\left(\operatorname{Im}(z)-\frac{5}{2} r\right) T} \int_{-T}^{-1} \mathrm{e}^{-\operatorname{Re}(z) x} d x \\
& +T^{\operatorname{Re}(w)-1} \mathrm{e}^{\left(\operatorname{Im}(z)+\frac{r}{2}\right) T} \int_{-T}^{-1} \mathrm{e}^{(-\operatorname{Re}(z)+100 r) x} d x
\end{aligned}
$$

Since for any fixed $A \in \mathbb{R}$ it holds that

$$
\begin{aligned}
& \int_{-T}^{-1} \mathrm{e}^{A x} d x \ll_{A} \begin{cases}1 & \text { if } A>0, \\
T & \text { if } A=0, \\
\mathrm{e}^{-A T} & \text { if } A<0\end{cases} \\
& \ll T\left(1+\mathrm{e}^{-A T}\right),
\end{aligned}
$$

we get

$$
\begin{aligned}
\int_{-T}^{-1} \ll_{r, z} T^{\operatorname{Re}(w)} \mathrm{e}^{\operatorname{Im}(z) T}\left(1+\mathrm{e}^{\left(\operatorname{Re}(z)-\tau^{(1)} r\right) T}\right) \\
\quad+T^{\operatorname{Re}(w)} \mathrm{e}^{\left(\operatorname{Im}(z)-\frac{5}{2} r\right) T}\left(1+\mathrm{e}^{\operatorname{Re}(z) T}\right) \\
\quad+T^{\operatorname{Re}(w)} \mathrm{e}^{\left(\operatorname{Im}(z)+\frac{r}{2}\right) T}\left(1+\mathrm{e}^{(\operatorname{Re}(z)-100 r) T}\right) .
\end{aligned}
$$

From the choice of $z$, this tends to zero as $N \rightarrow \infty$. Hence we get

$$
F_{r}(w, z)=R_{r}(w, z) \quad\left((w, z) \in D_{r}, \operatorname{Im}(z)<-r / 2\right)
$$

Equations (3.3) and (3.6) complete the proof.

\section{Revisit to Deninger's Theorems}

In this section we reprove Deninger's Theorem 1.1 from our method explained in Section 3. That is, we calculate both sides of Theorem 3.1 with $r=1$. We also reprove Deninger's Theorem 1.2. 


\section{1. $L_{1}^{(j)}$}

Lemma 4.1. Let $(w, z) \in D_{1}$ with $\operatorname{Im}(z)<-1 / 2$ and $\operatorname{Re}(w)>1$. Then we have

$$
\begin{aligned}
& L_{1}^{(1)}(w, z)=\sum_{\operatorname{Re}(\tau)>0} \frac{1}{(z+\tau)^{w}}-\frac{1}{\left(z+\frac{\mathrm{i}}{2}\right)^{w}} \\
& L_{1}^{(2)}(w, z)=\mathrm{e}^{\pi \mathrm{i} w} \sum_{\operatorname{Re}(\tau)>0} \frac{1}{(\tau-z)^{w}}+\mathrm{e}^{\pi \mathrm{i} w} \sum_{n=1}^{\infty} \frac{1}{\left(\left(2 n+\frac{1}{2}\right) \mathrm{i}-z\right)^{w}}
\end{aligned}
$$

where the argument lies in $(-\pi / 4,(3 \pi) / 4)$.

Proof. We treat $L_{1}^{(1)}(w, z)$. From the definition of $\theta^{*}(t)$ and $\theta(t)$ and Lemma 3.1 we have

$$
\begin{aligned}
L_{1}^{(1)}(w, z)= & \frac{1}{\Gamma(w)} \sum_{\operatorname{Re}(\tau)>0} \int_{0}^{\infty \mathrm{e}^{-\frac{\pi \mathrm{i}}{4}}} \mathrm{e}^{-(z+\tau) t} t^{w-1} d t \\
& -\frac{1}{\Gamma(w)} \int_{0}^{\infty \mathrm{e}^{-\frac{\pi \mathrm{i}}{4}}} \mathrm{e}^{-\left(z+\frac{\mathrm{i}}{2}\right) t} t^{w-1} d t \\
= & \sum_{\operatorname{Re}(\tau)>0} \frac{1}{(z+\tau)^{w}}-\frac{1}{\left(z+\frac{\mathrm{i}}{2}\right)^{w}} .
\end{aligned}
$$

Here to apply Lemma 3.1 we used $\operatorname{Re}(z+\tau)+\operatorname{Im}(z+\tau)>0$ and $\operatorname{Re}(z+$ $\left.\frac{\mathrm{i}}{2}\right)+\operatorname{Im}\left(z+\frac{\mathrm{i}}{2}\right)>0$, which follow from $\operatorname{Re}(z)+\operatorname{Im}(z)>-1 / 2, \operatorname{Im}(\tau)>$ $-1 / 2$ and $\tau^{(1)}>14$.

In the same manner as $L_{1}^{(1)}$, we obtain the formula for $L_{1}^{(2)}$.

Lemma 4.2. Let $-2<\operatorname{Re}(s)-\operatorname{Im}(s)<1, \operatorname{Re}(s)>1$ and $\operatorname{Re}(w)>1$. Then we have

$$
\begin{aligned}
& L_{1}^{(1)}\left(w,-\mathrm{i}\left(s-\frac{1}{2}\right)\right)=\mathrm{e}^{\frac{\pi \mathrm{i} w}{2}}\left(\sum_{\operatorname{Im}(\rho)<0} \frac{1}{(s-\rho)^{w}}-\frac{1}{(s-1)^{w}}\right) \\
& L_{1}^{(2)}\left(w,-\mathrm{i}\left(s-\frac{1}{2}\right)\right)=\mathrm{e}^{\frac{\pi \mathrm{i} w}{2}}\left(\sum_{\operatorname{Im}(\rho)>0} \frac{1}{(s-\rho)^{w}}+\sum_{n=1}^{\infty} \frac{1}{(s+2 n)^{w}}\right)
\end{aligned}
$$

where the argument lies in $(-\pi / 2, \pi / 2)$. The sums in (4.1) and (4.2) converge absolutely and uniformly on any compact subset of $\left\{(w, s) \in \mathbb{C}^{2}: \operatorname{Re}(w)>\right.$ $1, \operatorname{Re}(s)>1\}$. 
Proof. We put $z=-\mathrm{i}\left(s-\frac{1}{2}\right)$ in Lemma 4.1. We rewrite Lemma 4.1 in terms of $s$. We have

$$
\begin{aligned}
\sum_{\operatorname{Re}(\tau)>0} \frac{1}{(z+\tau)^{w}}= & \sum_{\operatorname{Re}(\tau)>0} \frac{1}{\left(-\mathrm{i}\left(s-\frac{1}{2}+\mathrm{i} \tau\right)\right)^{w}} \\
& : \arg \left(-\mathrm{i}\left(s-\frac{1}{2}+\mathrm{i} \tau\right)\right) \in\left(-\frac{\pi}{4}, \frac{3 \pi}{4}\right) \\
= & \mathrm{e}^{\frac{\pi \mathrm{i} w}{2}} \sum_{\operatorname{Re}(\tau)>0} \frac{1}{\left(s-\frac{1}{2}+\mathrm{i} \tau\right)^{w}} \\
& : \arg \left(s-\frac{1}{2}+\mathrm{i} \tau\right) \in\left(\frac{\pi}{4}, \frac{5 \pi}{4}\right) \\
= & \mathrm{e}^{\frac{\pi \mathrm{i} w}{2}} \sum_{\operatorname{Im}(\rho)<0} \frac{1}{(s-\rho)^{w}} .
\end{aligned}
$$

Here from the assumption $\operatorname{Re}(s)>1$ it holds that $\operatorname{Re}(s-\rho)>0$, i.e., $\arg (s-$ $\rho) \in(\pi / 4, \pi / 2) \subset(-\pi / 2, \pi / 2)$.

In the same manner we get

$$
\begin{aligned}
& \frac{1}{\left(z+\frac{\mathrm{i}}{2}\right)^{w}}=\frac{\mathrm{e}^{\frac{\pi \mathrm{i} w}{2}}}{(s-1)^{w}}, \quad \sum_{\operatorname{Re}(\tau)>0} \frac{1}{(\tau-z)^{w}}=\mathrm{e}^{-\frac{\pi \mathrm{i} w}{2}} \sum_{\operatorname{Im}(\rho)>0} \frac{1}{(s-\rho)^{w}} \\
& \sum_{n=1}^{\infty} \frac{1}{\left(\left(2 n+\frac{1}{2}\right) \mathrm{i}-z\right)^{w}}=\mathrm{e}^{-\frac{\pi \mathrm{i} w}{2}} \sum_{n=1}^{\infty} \frac{1}{(s+2 n)^{w}}
\end{aligned}
$$

where the argument lies in $(-\pi / 2, \pi / 2)$. Hence we obtain (4.1) and (4.2).

The absolute and locally uniform convergence easily follows from $\#\{\rho: \operatorname{Im}(\rho) \in(T, T+1], \zeta(\rho)=0\}=O(\log T)[19$, Theorem 9.2].

\section{2. $R_{1}$}

Lemma 4.3. Let $-2<\operatorname{Re}(s)-\operatorname{Im}(s)<1, \operatorname{Re}(s)>1$ and $\operatorname{Re}(w)>1$. Then we have

$$
R_{1}\left(w,-\mathrm{i}\left(s-\frac{1}{2}\right)\right)=-\frac{\mathrm{e}^{\frac{\pi \mathrm{i} w}{2}}}{\Gamma(w)} \sum_{p} \sum_{m=1}^{\infty} p^{-m s}(m \log p)^{w-1} \log p
$$

The sum converges absolutely and uniformly on any compact subset of $\left\{(w, s) \in \mathbb{C}^{2}: \operatorname{Re}(s)>1\right\}$. 
Proof. We calculate the residue in $R_{1}\left(w,-\mathrm{i}\left(s-\frac{1}{2}\right)\right)$ explicitly. From Lemma 2.2(1) (see also Remark 2.1) we have

$$
\begin{aligned}
& \operatorname{Res}_{t=\mathrm{i} m \log p} \mathrm{e}^{\mathrm{i}\left(s-\frac{1}{2}\right) t} \theta^{*}(t) t^{w-1} \\
& \quad=\operatorname{Res}_{t=\mathrm{i} m \log p} \mathrm{e}^{\mathrm{i}\left(s-\frac{1}{2}\right) t}\left(-\frac{t}{2 \pi \mathrm{i}} \mathrm{e}^{-\frac{\mathrm{i} t}{2}} \frac{p^{-m}}{m(t-\mathrm{i} m \log p)}\right) t^{w-1} \\
& =-\frac{\mathrm{e}^{\frac{\pi \mathrm{i} w}{2}}}{2 \pi \mathrm{i}} p^{-m s}(m \log p)^{w-1} \log p .
\end{aligned}
$$

Hence we obtain (4.3).

From the following estimate for any fixed $\varepsilon>0$ and $A \in \mathbb{R}$, we obtain the absolute and locally uniform convergence of the sum in (4.3):

$\sum_{p} \sum_{m=1}^{\infty} p^{-m(1+\varepsilon)}(m \log p)^{A} \log p=\sum_{n=2}^{\infty} \frac{\Lambda(n)}{n^{1+\varepsilon}}(\log n)^{A} \leq \sum_{n=2}^{\infty} \frac{(\log n)^{A+1}}{n^{1+\varepsilon}}<\infty$,

where $\Lambda$ is the von Mangoldt function. Hence we complete the proof.

\subsection{Reproof of Deninger's Theorems}

Proof of Deninger's Theorem 1.1. Applying Lemmas 4.2 and 4.3 to Theorem 3.1 with $r=1$, we obtain Deninger's Theorem 1.1 under the additional assumption $-2<\operatorname{Re}(s)-\operatorname{Im}(s)<1$. Since both sides are holomorphic in $\operatorname{Re}(s)>1$, we can remove it. This completes the proof of Deninger's Theorem 1.1.

Proof of Deninger's Theorem 1.2. We remark that each term on the lefthand side of Deninger's Theorem 1.1 has a meromorphic continuation to $w \in \mathbb{C}$ (see [9]). We also notice that the following Laurent expansion at $w=0$ holds:

$$
\frac{1}{\Gamma(w)}=w+O\left(w^{2}\right)
$$

Hence, taking the linear term of the Laurent expansion at $w=0$ in Deninger's Theorem 1.1, we obtain Deninger's Theorem 1.2.

\section{The double explicit formula}

In this section we prove Theorem 1.1 by calculating both sides of Theorem 3.1 with $r=2$ and we also prove Theorem 1.2. 


\section{1. $L_{2}^{(j)}$}

Lemma 5.1. Let $-4<\operatorname{Re}(s)-\operatorname{Im}(s)<2, \operatorname{Re}(s)>2$ and $\operatorname{Re}(w)>2$. Then we have

$$
\begin{aligned}
& L_{2}^{(1)}(w,-\mathrm{i}(s-1)) \\
& =\mathrm{e}^{\frac{\pi \mathrm{i} w}{2}}\left(\sum_{\operatorname{Im}\left(\rho_{1}\right)<0} \sum_{\operatorname{Im}\left(\rho_{2}\right)<0} \frac{1}{\left(s-\rho_{1}-\rho_{2}\right)^{w}}-2 \sum_{\operatorname{Im}(\rho)<0} \frac{1}{(s-1-\rho)^{w}}+\frac{1}{(s-2)^{w}}\right), \\
& L_{2}^{(2)}(w,-\mathrm{i}(s-1)) \\
& =-\mathrm{e}^{\frac{\pi \mathrm{i} w}{2}}\left(\sum_{\operatorname{Im}\left(\rho_{1}\right)>0} \sum_{\operatorname{Im}\left(\rho_{2}\right)>0} \frac{1}{\left(s-\rho_{1}-\rho_{2}\right)^{w}}+2 \sum_{\operatorname{Im}(\rho)>0} \sum_{n=1}^{\infty} \frac{1}{(s-\rho+2 n)^{w}}\right. \\
& \left.\quad+\sum_{n_{1}=1}^{\infty} \sum_{n_{2}=1}^{\infty} \frac{1}{\left(s+2 n_{1}+2 n_{2}\right)^{w}}\right),
\end{aligned}
$$

where the argument lies in $(-\pi / 2, \pi / 2)$. The sums converge absolutely and uniformly on any compact subset of $\left\{(w, s) \in \mathbb{C}^{2}: \operatorname{Re}(s)>2, \operatorname{Re}(w)>2\right\}$.

Proof. This is proved in the same manner as Section 4.1 without any difficulties.

\section{2. $R_{2}$}

Lemma 5.2. Let $-4<\operatorname{Re}(s)-\operatorname{Im}(s)<2, \operatorname{Re}(s)>2$ and $\operatorname{Re}(w)>2$. Then we have

$$
R_{2}(w,-\mathrm{i}(s-1))=\frac{\mathrm{e}^{\frac{\pi \mathrm{i} w}{2}}}{\Gamma(w)} \sum_{j=1}^{9} \widetilde{E}^{(j)}(w, s)
$$

where $\widetilde{E}^{(j)}(w, s):=E^{(j)}(w, s) \quad$ for $\quad j=1,2, \ldots, 7,9 \quad$ and $\quad \widetilde{E}^{(8)}(w, s):=$ $-E^{(8)}(w, s)$. Here $E^{(j)}(w, s)$ are defined as Theorem 1.1. 
Proof. Let $p$ be prime numbers and $m \in \mathbb{Z}_{\geq 1}$. Then, from Lemma 2.2(1) we have

$$
\begin{aligned}
\theta^{*}(t)^{2}= & \left(-\frac{t \mathrm{e}^{-\frac{\mathrm{i} t}{2}} p^{-m}}{2 \pi \mathrm{i} m(t-\mathrm{i} m \log p)}\right)^{2}+2\left(-\frac{t \mathrm{e}^{-\frac{\mathrm{i} t}{2}} p^{-m}}{2 \pi \mathrm{i} m(t-\mathrm{i} m \log p)}\right) \\
& \times\left(-\frac{t}{2 \pi \mathrm{i}} \mathrm{e}^{-\frac{\mathrm{i} t}{2}} \sum_{q} \sum_{\substack{n=1 \\
q^{n} \neq p^{m}}}^{\infty} \frac{q^{-n}}{n(t-\mathrm{i} n \log q)}+\sum_{j=2}^{8} A_{j}(t)\right) \\
& + \text { (holomorphic function near } t=\mathrm{i} m \log p) .
\end{aligned}
$$

Hence we get

$$
R_{2}(w,-\mathrm{i}(s-1))=\sum_{j=1}^{9} R_{2}^{(j)}(w, s),
$$

where

$$
\begin{aligned}
R_{2}^{(1)}(w, s):= & \frac{1}{2 \pi \mathrm{i} \Gamma(w)} \sum_{p} \sum_{m=1}^{\infty} \frac{p^{-2 m}}{m^{2}} \operatorname{Res}_{t=\mathrm{i} m \log p} \frac{\mathrm{e}^{\mathrm{i}(s-2) t} t^{w+1}}{(t-\mathrm{i} m \log p)^{2}}, \\
R_{2}^{(2)}(w, s):= & \frac{1}{\pi \mathrm{i} \Gamma(w)} \sum_{p} \sum_{m=1}^{\infty} \frac{p^{-m}}{m} \\
& \times \operatorname{Res}_{t=\mathrm{i} m \log p} \frac{\mathrm{e}^{\mathrm{i}(s-2) t} t^{w+1}}{t-\mathrm{i} m \log p}\left(\sum_{q} \sum_{\substack{n=1 \\
q^{n} \neq p^{m}}}^{\infty} \frac{q^{-n}}{n(t-\mathrm{i} n \log q)}\right), \\
R_{2}^{(j+1)}(w, s):= & -\frac{2}{\Gamma(w)} \sum_{p} \sum_{m=1}^{\infty} \frac{p^{-m}}{m} \operatorname{Res}_{t=\mathrm{i} m \log p} \frac{\mathrm{e}^{\mathrm{i}\left(s-\frac{3}{2}\right) t} t^{w}}{t-\mathrm{i} m \log p} A_{j}(t)
\end{aligned}
$$

for $j=2,3, \ldots, 8$. Calculating the residues, we obtain

$$
R_{2}^{(j)}(w, s)=\frac{\mathrm{e}^{\frac{\pi \mathrm{i} w}{2}}}{\Gamma(w)} \widetilde{E}^{(j)}(w, s)
$$

for any $j=1,2, \ldots, 9$. This completes the proof.

\subsection{Convergence of $E^{(j)}(w, s)$ : proof of Theorem 1.2}

In this subsection we prove Theorem 1.2, which is concerned with the absolute convergence of the sums and the integrals in $E^{(j)}(w, s)$ and $\widetilde{E}^{(j)}(w, s)$ for $j=1, \ldots, 9$. 
Proof of Theorem 1.2. From (4.4) we immediately obtain the desired convergence of the sums and the integrals in $E^{(j)}(w, s)$ except for $j=2,3,5$.

We treat the sum in $E^{(5)}(w, s)$. Applying the well-known asymptotic formula

$$
\frac{\Gamma^{\prime}}{\Gamma}(z)=\log z+O_{\varepsilon}\left(|z|^{-1}\right) \quad \text { as }|z| \rightarrow \infty,|\arg (z)| \leq \pi-\varepsilon
$$

and using (4.4), we get the desired convergence.

We deal with the sum in $E^{(3)}(w, s)$. Let $\varepsilon>0$ and $A<B$ be any fixed real numbers and suppose that $\operatorname{Re}(s) \geq 1+\varepsilon$ and $A \leq \operatorname{Re}(w) \leq B$. Then for any prime numbers $p, q$ and $m, n \in \mathbb{Z}_{\geq 1}$ we have

$$
\begin{aligned}
& \left|\frac{p^{-m s} q^{-n}(m \log p)^{w} \log p}{n(m \log p+n \log q)}\right| \\
& \quad \leq \begin{cases}\frac{2^{-(1+\varepsilon)} q^{-n}(\log 2)^{A+1}}{n^{2} \log q} & \text { if } p=2 \text { and } m=1, \\
\frac{p^{-m(1+\varepsilon)} q^{-n}(m \log p)^{B} \log p}{n^{2} \log q} & \text { otherwise. }\end{cases}
\end{aligned}
$$

From (2.2) we have

$$
\sum_{q} \sum_{n=1}^{\infty} \frac{2^{-(1+\varepsilon)} q^{-n}(\log 2)^{A+1}}{n^{2} \log q}<\infty
$$

From (2.2) and (4.4) we have

$$
\begin{aligned}
& \sum_{p} \sum_{\substack{m=1 \\
p^{m} \geq 3}}^{\infty} \sum_{q} \sum_{n=1}^{\infty} \frac{p^{-m(1+\varepsilon)} q^{-n}(m \log p)^{B} \log p}{n^{2} \log q} \\
& \quad \leq\left(\sum_{p} \sum_{m=1}^{\infty} p^{-m(1+\varepsilon)}(m \log p)^{B} \log p\right)\left(\sum_{q} \sum_{n=1}^{\infty} \frac{q^{-n}}{n^{2} \log q}\right) \\
& \quad<\infty
\end{aligned}
$$

Hence the sum in $E^{(3)}(w, s)$ converges absolutely and locally uniformly in $\left\{(w, s) \in \mathbb{C}^{2}: \operatorname{Re}(s)>1\right\}$.

We treat the sum in $E^{(2)}(w, s)$. Let $\varepsilon>0$ and $A<B$ be any fixed real numbers and suppose that $\operatorname{Re}(s) \geq 2+\varepsilon$ and $A \leq \operatorname{Re}(w) \leq B$. Then, for any 
prime numbers $p, q$ and $m, n \in \mathbb{Z}_{\geq 1}$ satisfying $q^{n} \neq p^{m}$, we have

$$
\begin{aligned}
& \left|\frac{p^{-m(s-1)} q^{-n}(m \log p)^{w} \log p}{n(m \log p-n \log q)}\right| \\
& \quad \leq \begin{cases}\frac{2^{-(1+\varepsilon)} q^{-n}(\log 2)^{A+1}}{n(n \log q-\log 2)} & \text { if } p=2 \quad \text { and } m=1, \\
\frac{p^{-m(1+\varepsilon)} q^{-n}(m \log p)^{B} \log p}{n|m \log p-n \log q|} & \text { otherwise. }\end{cases}
\end{aligned}
$$

We treat the sum on $p=2$ and $m=1$. From $\log x-\log 2 \geq\left(1-\frac{\log 2}{\log 3}\right)$ $\log x$ for any $x \geq 3$ and (2.2) we have

$$
\begin{aligned}
\sum_{q} \sum_{\substack{n=1 \\
q^{n} \geq 3}}^{\infty} \frac{2^{-(1+\varepsilon)} q^{-n}(\log 2)^{A+1}}{n(n \log q-\log 2)} & \leq \frac{2^{-(1+\varepsilon)}(\log 2)^{A+1}}{1-\frac{\log 2}{\log 3}} \sum_{q} \sum_{n=1}^{\infty} \frac{q^{-n}}{n^{2} \log q} \\
& <\infty
\end{aligned}
$$

Next, we treat the sum satisfying $(p, m) \neq(2,1)$. We have

$$
\begin{aligned}
& \sum_{p} \sum_{\substack{m=1 \\
p^{m} \geq 3}}^{\infty} \sum_{q} \sum_{\substack{n=1 \\
q^{n} \neq p^{m}}}^{\infty} \frac{p^{-m(1+\varepsilon)} q^{-n}(m \log p)^{B} \log p}{n|m \log p-n \log q|} \\
& \quad \leq \sum_{p} \sum_{m=1}^{\infty} \sum_{q} \sum_{\substack{n=1 \\
q^{n} \neq p^{m}}}^{\infty} \frac{p^{-m(1+\varepsilon)} q^{-n}(m \log p)^{B} \log p}{n|m \log p-n \log q|} \\
& \quad=\sum_{p} \sum_{m=1}^{\infty} \sum_{q} \sum_{\substack{n=1 \\
q^{n}<p^{m}}}^{\infty}+\sum_{p} \sum_{m=1}^{\infty} \sum_{q} \sum_{\substack{n=1 \\
p^{m}<q^{n}<p^{2 m}}}^{\infty}+\sum_{p} \sum_{m=1}^{\infty} \sum_{q} \sum_{\substack{n=1 \\
q^{n} \geq p^{2 m}}}^{\infty} .
\end{aligned}
$$

We treat the sum over $q^{n} \geq p^{2 m}$. Then, since $2 m \log p \leq n \log q$, we have $n \log q-m \log p \geq(n \log q) / 2$. Hence together with (2.2) and (4.4) we get

$$
\begin{aligned}
\sum_{p} \sum_{m=1}^{\infty} \sum_{q} \sum_{\substack{n=1 \\
q^{n} \geq p^{2 m}}}^{\infty} & \leq 2\left(\sum_{p} \sum_{m=1}^{\infty} p^{-m(1+\varepsilon)}(m \log p)^{B} \log p\right)\left(\sum_{q} \sum_{n=1}^{\infty} \frac{q^{-n}}{n^{2} \log q}\right) \\
& <\infty .2
\end{aligned}
$$


Next we deal with the sum over $p^{m}<q^{n}<p^{2 m}$. From Lemma 2.5 we have

$$
\begin{aligned}
& \sum_{q} \sum_{\substack{n=1 \\
p^{m}<q^{n}<p^{2 m}}}^{\infty} \frac{q^{-n}}{n|m \log p-n \log q|} \\
& \quad \leq \sum_{q} \sum_{\substack{n=1 \\
p^{m}<q^{n}<p^{2 m}}}^{\infty} q^{-n} \frac{q^{n}}{q^{n}-p^{m}} \leq \sum_{l=1}^{p^{2 m}-p^{m}-1} \frac{1}{\left(p^{m}+l\right)-p^{m}} \ll m \log p .
\end{aligned}
$$

Hence together with (4.4) we get

$$
\sum_{p} \sum_{m=1}^{\infty} \sum_{q} \sum_{\substack{n=1 \\ p^{m}<q^{n}<p^{2 m}}}^{\infty} \ll \sum_{p} \sum_{m=1}^{\infty} p^{-m(1+\varepsilon)}(m \log p)^{B+1} \log p<\infty
$$

Next we treat the sum over $q^{n}<p^{m}$. From Lemma 2.5 we have

$$
\begin{aligned}
& \sum_{q} \sum_{\substack{n=1 \\
q^{n}<p^{m}}}^{\infty} \frac{q^{-n}}{n|m \log p-n \log q|} \\
& \quad \leq \sum_{q} \sum_{\substack{n=1 \\
q^{n}<p^{m}}}^{\infty} q^{-n} \frac{p^{m}}{p^{m}-q^{n}} \leq p^{m} \sum_{l=1}^{p^{m}-1} \frac{1}{l\left(p^{m}-l\right)}=\sum_{l=1}^{p^{m}-1} \frac{1}{l}+\sum_{l=1}^{p^{m}-1} \frac{1}{p^{m}-l}
\end{aligned}
$$

$\ll m \log p$.

Hence together with (4.4) we get

$$
\sum_{p} \sum_{m=1}^{\infty} \sum_{q} \sum_{\substack{n=1 \\ q^{n}<p^{m}}}^{\infty} \ll \sum_{p} \sum_{m=1}^{\infty} p^{-m(1+\varepsilon)}(m \log p)^{B+1} \log p<\infty .
$$

From (5.2) to (5.4), (5.1) converges. This completes the proof.

\subsection{Proof of Theorem 1.1}

Proof of Theorem 1.1. Assume that $(w, s)$ satisfies the assumption of Lemma 5.1. Applying Lemmas 5.1 and 5.2 to Theorem 3.1 with $r=2$, 
we have

$$
\begin{aligned}
& -\sum_{\operatorname{Im}\left(\rho_{1}\right)>0} \sum_{\operatorname{Im}\left(\rho_{2}\right)>0} \frac{1}{\left(s-\rho_{1}-\rho_{2}\right)^{w}}+\sum_{\operatorname{Im}\left(\rho_{1}\right)<0} \sum_{\operatorname{Im}\left(\rho_{2}\right)<0} \frac{1}{\left(s-\rho_{1}-\rho_{2}\right)^{w}} \\
& -2 \sum_{\operatorname{Im}(\rho)<0} \frac{1}{(s-1-\rho)^{w}}-2 \sum_{\operatorname{Im}(\rho)>0} \sum_{n=1}^{\infty} \frac{1}{(s-\rho+2 n)^{w}} \\
& +\frac{1}{(s-2)^{w}}-\sum_{n_{1}=1}^{\infty} \sum_{n_{2}=1}^{\infty} \frac{1}{\left(s+2 n_{1}+2 n_{2}\right)^{w}} \\
& =\frac{1}{\Gamma(w)} \sum_{j=1}^{9} \widetilde{E}^{(j)}(w, s) .
\end{aligned}
$$

From Deninger's Theorem 1.1 we have

$$
\frac{E^{(8)}(w, s)}{\Gamma(w)}=\sum_{\rho} \frac{1}{(s-1-\rho)^{w}}-\frac{1}{(s-2)^{w}}+\sum_{n=1}^{\infty} \frac{1}{(s+2 n-1)^{w}} .
$$

Doubling this and adding it to (5.5), we get the desired result under the extra assumption $-4<\operatorname{Re}(s)-\operatorname{Im}(s)<2$. Since both sides are holomorphic with respect to $s$ in $\operatorname{Re}(s)>2$ owing to Theorem 1.2, we can remove it. This completes the proof.

\section{Euler product expressions for $\zeta^{\otimes 2}(s)$}

In this section we prove Theorem 1.3.

Proof of Theorem 1.3. We remark that each term on the left-hand side of Theorem 1.1 has a meromorphic continuation to all $w \in \mathbb{C}$. (See [9].) Hence, taking the coefficient of the linear term of the Laurent expansion at $w=0$ in Theorem 1.1 (see also (4.5)), we obtain the desired result.

\section{Discussion}

In this section we raise a problem (Proposition 7.1) when we try to analyze the nontrivial zeros of the Riemann zeta function via our results.

Since it is difficult to treat sums over the prime numbers, we rewrite the right-hand side of Theorems 1.1 and 1.3 by sums over the zeros and the pole of the Riemann zeta function as much as possible. From Deninger's Theorem 
1.1 we have

$$
\begin{aligned}
\frac{E^{(9)}(w, s)}{\Gamma(w)}= & -2 \sum_{\rho} \sum_{n=1}^{\infty} \frac{1}{(s+2 n-\rho)^{w}}+2 \sum_{n=1}^{\infty} \frac{1}{(s-1+2 n)^{w}} \\
& -2 \sum_{n_{1}=1}^{\infty} \sum_{n_{2}=1}^{\infty} \frac{1}{\left(s+2 n_{1}+2 n_{2}\right)^{w}}
\end{aligned}
$$

Applying this and (5.6) to Theorem 1.1, we have

Lemma 7.1. In $\operatorname{Re}(s)>2$ and $\operatorname{Re}(w)>2$ we have

$$
\begin{aligned}
& -\sum_{\operatorname{Im}\left(\rho_{1}\right)>0} \sum_{\operatorname{Im}\left(\rho_{2}\right)>0} \frac{1}{\left(s-\rho_{1}-\rho_{2}\right)^{w}}+\sum_{\operatorname{Im}\left(\rho_{1}\right)<0} \sum_{\operatorname{Im}\left(\rho_{2}\right)<0} \frac{1}{\left(s-\rho_{1}-\rho_{2}\right)^{w}} \\
& +\sum_{\operatorname{Im}(\rho)>0} \frac{1}{(s-1-\rho)^{w}}-\sum_{\operatorname{Im}(\rho)<0} \frac{1}{(s-1-\rho)^{w}}-\sum_{n=1}^{\infty} \frac{1}{(s-1+2 n)^{w}} \\
& +2 \sum_{\operatorname{Im}(\rho)<0} \sum_{n=1}^{\infty} \frac{1}{(s+2 n-\rho)^{w}}+\sum_{n_{1}=1}^{\infty} \sum_{n_{2}=1}^{\infty} \frac{1}{\left(s+2 n_{1}+2 n_{2}\right)^{w}} \\
& =\frac{1}{\Gamma(w)} \sum_{j=1}^{7} E^{(j)}(w, s),
\end{aligned}
$$

where $E^{(j)}(w, s)$ are defined as Theorem 1.1.

Differentiating Lemma 7.1 with respect to $w$ at $w=0$, we obtain

Lemma 7.2. In $\operatorname{Re}(s)>2$ we have

$$
\begin{gathered}
\frac{\prod_{\operatorname{Im}\left(\rho_{j}\right)>0}\left(s-\rho_{1}-\rho_{2}\right) \prod_{\operatorname{Im}(\rho)<0}(s-1-\rho)}{\prod_{\operatorname{Im}\left(\rho_{j}\right)<0}\left(s-\rho_{1}-\rho_{2}\right) \prod_{\operatorname{Im}(\rho)>0}(s-1-\rho)\left(\prod_{\substack{\operatorname{Im}(\rho)<0, n \geq 1}}(s+2 n-\rho)\right)^{2}} \\
\times \prod_{\prod_{n=1}^{\infty}\left(s+2 n_{1}+2 n_{2}\right)}^{\infty}(s+2 n-1)
\end{gathered}
$$




$$
=\exp \left[\sum_{j=1}^{7} E^{(j)}(s)\right],
$$

where $E^{(j)}(s)$ are defined as Theorem 1.3.

We note that (7.1) has zeros or poles at $s=\rho_{1}+\rho_{2}$ if $\rho_{1}+\rho_{2} \neq 1+\rho^{\prime}$ for any nontrivial zeros $\rho^{\prime}$ of $\zeta(s)$. In particular, $s=2 \rho$ are zeros or poles if $2 \rho \neq 1+\rho^{\prime}$ for any $\rho^{\prime}$. Therefore, in view of the zero-free region of the Riemann zeta function, it is interesting to improve the absolutely convergent region (Theorem 1.2 with $w=0$ ) for the right-hand side of (7.1). If there would exist $\vartheta>0$ such that the sums and the integrals in $E^{(j)}(s)$ converge absolutely for $\operatorname{Re}(s)>2-\vartheta$ for any $j=1, \ldots, 7$, we might improve the zerofree region of the Riemann zeta function. But when we consider $E^{(j)}(s)$ separately, it is impossible because the holomorphy of $E^{(2)}(s)$ and $E^{(6)}(s)$ is broken at $s=2$ as follows:

\section{Proposition 7.1.}

$$
E^{(2)}(s)-\frac{1}{2 \pi \mathrm{i}}(\log (s-2))^{2}+\frac{1}{\pi \mathrm{i}} \log ((s-2) \zeta(s-1)) \log (s-2)
$$

has an analytic continuation as a holomorphic function to

$$
\begin{aligned}
D_{1}:= & \{s \in \mathbb{C}: \operatorname{Re}(s)>1\} \\
& \backslash\left(\left\{\rho_{1}+\rho_{2}+\lambda: \operatorname{Im}\left(\rho_{1}\right) \times \operatorname{Im}\left(\rho_{2}\right)>0, \zeta\left(\rho_{j}\right)=0, \lambda \leq 0\right\}\right. \\
& \cup\{\rho+\lambda: 0<\operatorname{Re}(\rho)<1, \zeta(\rho)=0, \lambda \leq 1\}),
\end{aligned}
$$

where in the initial domain $\operatorname{Re}(s)>2$, the above logarithms are taken as $\log (s-2), \log ((s-2) \zeta(s-1)) \in \mathbb{R}$ if $s>2$ and extending them analytically to $\operatorname{Re}(s)>2$.

$$
E^{(6)}(s)+\frac{1}{2 \pi \mathrm{i}}(\log (s-2))^{2}-\frac{1}{\pi \mathrm{i}} \log ((s-2) \zeta(s-1)) \log (s-2)
$$

has an analytic continuation as a holomorphic function to

$$
D_{2}:=\mathbb{C} \backslash(\{\rho+\lambda: 0<\operatorname{Re}(\rho)<1, \zeta(\rho)=0, \lambda \leq 1\} \cup\{s \in \mathbb{R}: s \leq 1\})
$$


In particular, (7.2) and (7.3) have an analytic continuation to a region including $\operatorname{Re}(s) \geq 2$.

Proof. We show (2). ${ }^{3}$ We have

$$
\begin{aligned}
E^{(6)}(s)= & \frac{1}{\pi \mathrm{i}} \int_{0}^{1} \frac{\zeta^{\prime}}{\zeta}(s-u) \log (-\zeta(u)) d u \\
= & \frac{1}{\pi \mathrm{i}} \int_{0}^{1}\left(\frac{\zeta^{\prime}}{\zeta}(s-u)+\frac{1}{s-u-1}\right) \log (-\zeta(u)) d u \\
& -\frac{1}{\pi \mathrm{i}} \int_{0}^{1} \frac{\log (-\zeta(u))}{s-u-1} d u \\
= & : A(s)+B(s) .
\end{aligned}
$$

We first investigate an analyticity of $A(s)$. Since $\frac{\zeta^{\prime}}{\zeta}(z)+\frac{1}{z-1}$ is holomorphic in $\mathbb{C} \backslash\left(\{\rho \in \mathbb{C}: 0<\operatorname{Re}(\rho)<1, \zeta(\rho)=0\} \cup\left\{-2 n: n \in \mathbb{Z}_{\geq 1}\right\}\right), \quad \zeta(s-u)+$ $\frac{1}{s-u-1}$ is uniformly bounded on $(s, u) \in K \times[0,1]$ for any compact subset $K$ of

$$
\begin{aligned}
& D_{3}:= \mathbb{C} \backslash(\{\rho+\lambda: 0<\operatorname{Re}(\rho)<1, \zeta(\rho)=0,0 \leq \lambda \leq 1\} \\
&\left.\cup\left\{-2 n+\lambda: n \in \mathbb{Z}_{\geq 1}, 0 \leq \lambda \leq 1\right\}\right) .
\end{aligned}
$$

This, together with that $\log (-\zeta(u))$ is absolutely integrable in $[0,1]$, implies that $A(s)$ has an analytic continuation to $D_{3}$.

We consider $B(s)$. We divide $B(s)$ as

$$
B(s)=-\frac{1}{\pi \mathrm{i}} \int_{0}^{1} \frac{\log ((u-1) \zeta(u))}{s-u-1} d u+\frac{1}{\pi \mathrm{i}} \int_{0}^{1} \frac{\log (1-u)}{s-u-1} d u=: B_{1}(s)+B_{2}(s) .
$$

We treat $B_{2}(s)$. We temporally restrict $s$ to $|s-2|>1$ in addition to $\operatorname{Re}(s)>$ 2. Then we have

$$
\begin{aligned}
B_{2}(s) & =\frac{1}{\pi \mathrm{i}} \int_{0}^{1} \frac{\log u}{s-2+u} d u=\frac{1}{\pi \mathrm{i}(s-2)} \int_{0}^{1}\left(\sum_{n=0}^{\infty}\left(\frac{u}{2-s}\right)^{n}\right) \log u d u \\
& =\frac{1}{\pi \mathrm{i}} \sum_{n=0}^{\infty} \frac{1}{(n+1)^{2}}\left(\frac{1}{2-s}\right)^{n+1}=\frac{1}{\pi \mathrm{i}} \operatorname{Li}_{2}\left(\frac{1}{2-s}\right),
\end{aligned}
$$

where $\operatorname{Li}_{2}(z):=\sum_{n=1}^{\infty} \frac{z^{n}}{n^{2}}$ is the dilogarithm. It is well-known that $\operatorname{Li}_{2}(z)$ is originally defined in $|z|<1$, has an analytic continuation to $z \in \mathbb{C} \backslash[1, \infty)$

\footnotetext{
${ }^{3}$ The method of the proof is originated in [6, pp. 116-117], which analyzed the behavior of the final term in Lemma 2.1(1) near $t=0$.
} 
and satisfies the transformation formula

(7.4)

$$
\operatorname{Li}_{2}\left(\frac{1}{z}\right)=-\operatorname{Li}_{2}(z)-\frac{\pi^{2}}{6}-\frac{1}{2}(\log (-z))^{2} \quad(z \in \mathbb{C} \backslash[0, \infty),|\arg (-z)|<\pi) .
$$

Putting $z=2-s$ in (7.4), we get

$$
B_{2}(s)=-\frac{1}{2 \pi \mathrm{i}}(\log (s-2))^{2}-\frac{1}{\pi \mathrm{i}} \operatorname{Li}_{2}(2-s)+\frac{\pi \mathrm{i}}{6} .
$$

This implies that $B_{2}(s)+\frac{1}{2 \pi \mathrm{i}}(\log (s-2))^{2}$ has an analytic continuation to $D_{4}:=\mathbb{C} \backslash(-\infty, 1]$. We deal with $B_{1}(s)$. We restrict $s$ to $|s-2|<1$ in addition to $\operatorname{Re}(s)>2$. Since $\log ((z-1) \zeta(z))$ is holomorphic in $|z-1|<3$ and $[\log ((z-1) \zeta(z))]_{z=1}=0$, it has the Taylor expansion of the following form:

$$
\log ((z-1) \zeta(z))=\sum_{n=1}^{\infty} a_{n}(z-1)^{n}
$$

which converges absolutely and locally uniformly in $|z-1|<3$. Hence we have

$$
B_{1}(s)=-\frac{1}{\pi \mathrm{i}} \sum_{n=1}^{\infty} a_{n} \int_{0}^{1} \frac{(u-1)^{n}}{s-u-1} d u
$$

Applying the binomial theorem to $(u-1)^{n}=((u+1-s)+(s-2))^{n}$, we have

$$
\begin{aligned}
B_{1}(s)= & -\frac{1}{\pi \mathrm{i}} \sum_{n=1}^{\infty} a_{n} \sum_{k=0}^{n}\left(\begin{array}{l}
n \\
k
\end{array}\right)(-1)^{k}(s-2)^{n-k} \int_{0}^{1}(s-u-1)^{k-1} d u \\
= & -\frac{1}{\pi \mathrm{i}} \sum_{n=1}^{\infty} a_{n}(s-2)^{n} \int_{0}^{1} \frac{d u}{s-u-1} \\
& -\frac{1}{\pi \mathrm{i}} \sum_{n=1}^{\infty} a_{n} \sum_{k=1}^{n}\left(\begin{array}{l}
n \\
k
\end{array}\right)(-1)^{k}(s-2)^{n-k} \int_{0}^{1}(s-u-1)^{k-1} d u .
\end{aligned}
$$

By (7.5), the first term in (7.6) equals

$$
-\frac{1}{\pi \mathrm{i}} \log ((s-2) \zeta(s-1))(-\log (s-2)+\log (s-1)),
$$


where $|\arg (s-2)|,|\arg (s-1)|<\pi / 2$. Hence we get

$$
\begin{aligned}
B_{1}(s) & -\frac{1}{\pi \mathrm{i}} \log ((s-2) \zeta(s-1)) \log (s-2) \\
= & -\frac{1}{\pi \mathrm{i}} \log ((s-2) \zeta(s-1)) \log (s-1) \\
& \quad-\frac{1}{\pi \mathrm{i}} \sum_{n=1}^{\infty} a_{n} \sum_{k=1}^{n}\left(\begin{array}{l}
n \\
k
\end{array}\right)(-1)^{k}(s-2)^{n-k} \int_{0}^{1}(s-u-1)^{k-1} d u
\end{aligned}
$$

We investigate the analyticity of (7.7). Clearly, the left-hand side of (7.7) can be extended analytically to

$$
D_{5}:=\mathbb{C} \backslash(\{\rho+\lambda: 0<\operatorname{Re}(\rho)<1, \zeta(\rho)=0, \lambda \leq 1\} \cup\{s \leq 2\}) .
$$

Next we observe (7.7) near $s=2$. We easily see that the first term on the right-hand side of (7.7) is holomorphic in $|s-2|<1$. To consider the second term, we take any fixed $\varepsilon \in(0,1)$ and suppose $|s-2| \leq 1-\varepsilon$. Then, for any $u \in[0,1], n \in \mathbb{Z}_{\geq 1}$ and $k \in \mathbb{Z}$ satisfying $1 \leq k \leq n$, we have

$$
\begin{aligned}
\left|a_{n}\left(\begin{array}{l}
n \\
k
\end{array}\right)(-1)^{k}(s-2)^{n-k}(s-u-1)^{k-1}\right| & =\left|a_{n}\right|\left(\begin{array}{l}
n \\
k
\end{array}\right)|s-2|^{n-k}|s-2+1-u|^{k-1} \\
& \leq\left|a_{n}\right|\left(\begin{array}{l}
n \\
k
\end{array}\right)(1-\varepsilon)^{n-k}(2-\varepsilon)^{k-1} .
\end{aligned}
$$

Since the sum in (7.5) converges absolutely in $|z-1|<3$, we have

$$
\begin{aligned}
& \sum_{n=1}^{\infty} \sum_{k=1}^{n} \int_{0}^{1}\left|a_{n}\right|\left(\begin{array}{l}
n \\
k
\end{array}\right)(1-\varepsilon)^{n-k}(2-\varepsilon)^{k-1} d u \\
& \quad \leq \sum_{n=1}^{\infty}\left|a_{n}\right| \sum_{k=0}^{n}\left(\begin{array}{l}
n \\
k
\end{array}\right)(1-\varepsilon)^{n-k}(2-\varepsilon)^{k-1} \\
& \quad=\frac{1}{2-\varepsilon} \sum_{n=1}^{\infty}\left|a_{n}\right|(3-2 \varepsilon)^{n}<\infty .
\end{aligned}
$$

Equations (7.8) and (7.9) imply that the sums and the integral in the second term converge absolutely and locally uniformly in $|s-2|<1$. In particular, the second term can be extended analytically in $|s-2|<1$. Hence $B_{1}(s)-$ $\frac{1}{\pi \mathrm{i}} \log ((s-2) \zeta(s-1)) \log (s-2)$ can be extended analytically to $D_{6}:=D_{5} \cup$ $\{s \in \mathbb{C}:|s-2|<1\}=\mathbb{C} \backslash(\{\rho+\lambda: 0<\operatorname{Re}(\rho)<1, \zeta(\rho)=0, \lambda \leq 1\} \cup\{s \leq 1\})$. 
From the above observations,

$$
\begin{aligned}
E^{(6)}(s) & +\frac{1}{2 \pi \mathrm{i}}(\log (s-2))^{2}-\frac{1}{\pi \mathrm{i}} \log ((s-2) \zeta(s-1)) \log (s-2) \\
= & \left.A(s)+\left(B_{1}(s)-\frac{1}{\pi \mathrm{i}} \log ((s-2) \zeta(s-1)) \log (s-2)\right)\right) \\
& +\left(B_{2}(s)+\frac{1}{2 \pi \mathrm{i}}(\log (s-2))^{2}\right)
\end{aligned}
$$

has an analytic continuation to $D_{3} \cap D_{4} \cap D_{6}=D_{2}$. This implies (2).

To show (1), we take a logarithm of (7.1). Since the left-hand side has neither zeros nor poles in $D_{1}, \log ($ the left-hand side of $(7.1))$ is holomorphic in $D_{1}$. On the other hand, from Theorem 1.2 with $w=0, E^{(j)}(s)$ are holomorphic in $D_{1}$ except for $j=2,6$. These imply that

$$
\begin{aligned}
E^{(2)} & (s)+E^{(6)}(s) \\
= & \left(E^{(2)}(s)-\frac{1}{2 \pi \mathrm{i}}(\log (s-2))^{2}+\frac{1}{\pi \mathrm{i}} \log ((s-2) \zeta(s-1)) \log (s-2)\right) \\
& +\left(E^{(6)}(s)+\frac{1}{2 \pi \mathrm{i}}(\log (s-2))^{2}-\frac{1}{\pi \mathrm{i}} \log ((s-2) \zeta(s-1)) \log (s-2)\right)
\end{aligned}
$$

is also holomorphic in $D_{1}$. From Proposition 7.1(2), the second brace is holomorphic in $D_{1}$. This completes the proof.

\section{Acknowledgment}

The author is supported by JSPS Research Fellowships for Young Scientists (DC1).

\section{References}

[1] H. Akatsuka, Euler product expression of triple zeta functions, Internat. J. Math. 16 (2005), 111-136.

[2] H. Akatsuka, Functional equations for absolute tensor products, J. Ramanujan Math. Soc. 20 (2005), 91-105.

[3] H. Akatsuka, Degeneration of multiple Euler products, Acta Arith. 121 (2006), 63-91. 
[4] H. Akatsuka, Absolute tensor products, Kummer's formula and functional equations for multiple Hurwitz zeta functions, Acta Arith. 125 (2006), 63-78.

[5] K. Barner, On A. Weil's explicit formula, J. Reine Angew. Math. 323 (1981), 139-152.

[6] H. Cramér, Studien über die Nullstellen der Riemannschen Zetafunktion, Math. Z. 4 (1919), 104-130.

[7] C. Deninger, Local L-factors of motives and regularized determinants, Invent. Math. 107 (1992), 135-150.

[8] A. P. Guinand, Fourier reciprocities and the Riemann zeta-function, Proc. London Math. Soc. (2) 51 (1950), 401-414.

[9] M. Hirano, N. Kurokawa and M. Wakayama, Half zeta functions, J. Ramanujan Math. Soc. 18 (2003), 195-209.

[10] G. Illies, Regularized products and determinants, Comm. Math. Phys. 220 (2001), 69-94.

[11] N. Kurokawa, Multiple zeta functions: an example, Adv. Stud. Pure Math. 21 (1992), 219-226.

[12] S. Koyama and N. Kurokawa, Multiple zeta functions: the double sine function and the signed double Poisson summation formula, Compos. Math. 140 (2004), 1176-1190.

[13] S. Koyama and N. Kurokawa, Multiple Euler products (in Russian), Proc. St. Petersburg Math. Soc. 11 (2005), 123-166. English transl.: Amer. Math. Soc. Transl. Ser. 218 (2006), 101-140.

[14] N. Kurokawa and M. Wakayama, Absolute tensor products, Int. Math. Res. Not. 2004(5) (2004), 249-260.

[15] Y. I. Manin, Lectures on zeta functions and motives (according to Deninger and Kurokawa), Astérisque 228 (1995), 121-163.

[16] A. Narukawa, The modular properties and the integral representations of the multiple elliptic gamma functions, Adv. Math. 189 (2004), $247-267$.

[17] M. Schröter, Über Kurokawa-Tensorprodukte von L-Reihen, Schriftenreihe Math. Insti. Univ. Münster 3 Ser. Heft 18 (1996), ii+240pp. 
[18] M. Schröter and C. Soulé, On a result of Deninger concerning Riemann's zeta function, Proc. Sympos. Pure Math. 55 (1994), 745-747.

[19] E. Titchmarsh, The theory of the Riemann zeta-function, 2nd ed., The Clarendon Press, Oxford University Press, 1986.

[20] A. Weil, Sur les "formules explicites" de la théorie des nombres premiers, Comm. Sém. Math. Univ. Lund [Medd. Lunds Univ. Mat. Sem.] 1952 (1952), Tome Supplementaire 252-265. [Collected papers. Vol. II (1951-1964), 48-61.]

Department of Mathematics

Tokyo Institute of TeChNology

2-12-1 Ookayama, Meguro-Ku

TOKYO, 152-8551

JAPAN

E-mail address: akatsuka@math.titech.ac.jp

Received OCtober 5, 2009 
